\title{
Tonotopic Organization in Human Auditory Cortex Revealed by Progressions of Frequency Sensitivity
}

\author{
Thomas M. Talavage, ${ }^{1-3}$ Martin I. Sereno, ${ }^{4}$ Jennifer R. Melcher, ${ }^{1,5,6}$ Patrick J. Ledden, ${ }^{2}$ \\ Bruce R. Rosen, ${ }^{2}$ and Anders M. Dale ${ }^{2}$ \\ ${ }^{1}$ Speech and Hearing Sciences Program, Massachusetts Institute of Technology-Harvard Division of Health Sciences and Technology, \\ Cambridge, Massachusetts 02139; ${ }^{2}$ Massachusetts General Hospital-Nuclear Magnetic Resonance Center, Department of Radiology, \\ Massachusetts General Hospital, Boston, Massachusetts 02129; ${ }^{3}$ Electrical and Computer Engineering/Biomedical Engineering, \\ Purdue University, West Lafayette, Indiana 47907-2035; ${ }^{4}$ Cognitive Science Department, University of California-San Diego, \\ La Jolla, California 92093; ${ }^{5}$ Department of Otology and Laryngology, Harvard Medical School, Boston, Massachusetts 02115; and \\ ${ }^{6}$ Department of Otolaryngology, Massachusetts Eye and Ear Infirmary, Boston, Massachusetts 02114
}

Submitted 13 December 2002; accepted in final form 7 November 2003

Talavage, Thomas M., Martin I. Sereno, Jennifer R. Melcher, Patrick J. Ledden, Bruce R. Rosen, and Anders M. Dale. Tonotopic organization in human auditory cortex revealed by progressions of frequency sensitivity. J Neurophysiol 91: 1282-1296, 2004. First published November 12, 2003; 10.1152/jn.01125.2002. Functional neuroimaging experiments have revealed an organization of frequencydependent responses in human auditory cortex suggestive of multiple tonotopically organized areas. Numerous studies have sampled cortical responses to isolated narrow-band stimuli, revealing multiple locations in auditory cortex at which the position of response varies systematically with frequency content. Because appropriate anatomical or functional grouping of these distinct frequency-dependent responses is uncertain, the number and location of tonotopic mappings within human auditory cortex remains unclear. Further, sampling does not address whether the observed mappings exhibit continuity as a function of position. This functional magnetic resonance imaging study used frequency-swept stimuli to identify progressions of frequency sensitivity across the cortical surface. The center-frequency of narrow-band, amplitude-modulated noise was slowly swept between 125 and $8,000 \mathrm{~Hz}$. The latency of response relative to sweep onset was determined for each cortical surface location. Because frequency varied systematically with time, response latency indicated the frequency to which a location was maximally sensitive. Areas of cortex exhibiting a progressive change in response latency with position were considered tonotopically organized. There exist two main findings. First, six progressions of frequency sensitivity (i.e., tonotopic mappings) were repeatably observed in the superior temporal plane. Second, the locations of the higher- and lower-frequency endpoints of these progressions were approximately congruent with regions reported to be most responsive to discrete higher- and lower-frequency stimuli. Based on these findings and previous anatomical work, we propose a correspondence between these progressions and anatomically defined cortical areas, suggesting that five areas in human auditory cortex exhibit at least six tonotopic organizations.

\section{N T R O D U C T I O N}

Tonotopy is a fundamental organizing principle of the auditory system. Tonotopy arises from mechanical properties of the cochlea and is apparent as a linear arrangement of neurons according to best frequency (BF, the acoustic frequency to which a neuron is most sensitive). In the cerebral cortex,

Address for reprint requests and other correspondence: T. M. Talavage, 465 Northwestern Ave., Purdue University, West Lafayette, IN 47907-2035 (E-mail: tmt@ecn.purdue.edu). tonotopy is seen as a progressive change in neuronal best frequency with position along the cortical surface-a tonotopic map. Multiple tonotopic maps have been observed in the auditory cortex of many animal species. For instance, as many as seven tonotopic mappings have been observed in the cat cortex, each corresponding to a cytoarchitecturally distinct area (e.g., Heil et al. 1994; Reale and Imig 1980). Four or more tonotopic mappings, also corresponding to cytoarchitectural divisions of the auditory cortex, have been observed in the monkey brain (e.g., Morel et al. 1993). Many of these mappings abut at their high- or low-frequency extremes and exhibit opposing spatial gradients of frequency sensitivity. The resulting "mirror-image" organization between adjacent tonotopic mappings resembles the arrangement of retinotopic fields in the visual cortex (e.g., Sereno et al. 1995).

The tonotopic organization of human auditory cortex-located on the superior surface of the temporal lobe-remains poorly understood. However, a degree of tonotopic organization and the presence of some major organizational elements seen in animals have been demonstrated by previous human studies. These studies have localized cortical responses to multiple discrete, narrow-band stimuli having different center frequencies. Cortical activation studies using such probes have been conducted using various modalities, including magnetoencephalography (MEG) (Cansino et al. 1994; Pantev et al. 1988, 1994; Romani et al. 1982a,b), positron emission tomography (PET) (Lauter et al. 1985; Lockwood et al. 1999), microelectrode recordings (Howard et al. 2000), and functional magnetic resonance imaging (fMRI) (Bilecen et al. 1998; Schönwiesner et al. 2002; Talavage et al. 2000; Wessinger et al. 1997; Yang et al. 2000). Studies examining the frequency organization of human auditory cortex may be divided into two groups, those using two stimulus frequencies and those using more than two.

Studies using two stimulus frequencies have demonstrated that the cortical location of peak response is frequency dependent. Lauter et al. (1985), Wessinger et al. (1997), Bilecen et al. (1998), and Lockwood et al. (1999) demonstrated gross selectivity in the auditory cortex for two disparate tonal frequen-

The costs of publication of this article were defrayed in part by the payment of page charges. The article must therefore be hereby marked "advertisement" in accordance with 18 U.S.C. Section 1734 solely to indicate this fact. 
cies- 55 and $880 \mathrm{~Hz}$ for Wessinger et al., 500 and 4,000 Hz for the others-by comparing the cortical activity during tone presentation with activity during "no stimulus" conditions. Talavage et al. (2000) directly compared cortical activity during the presentation of two stimuli (tone bursts, music, noise bursts) limited to frequencies $<660 \mathrm{~Hz}$ (low) and $>2,490 \mathrm{~Hz}$ (high). This study consistently demonstrated eight regions of response, each exhibiting a greater sensitivity to either loweror higher-frequency stimulation.

Studies that used more than two acoustic frequencies generally observed a covariance of position of response with stimulus frequency (Cansino et al. 1994; Pantev et al. 1988, 1994; Romani et al. 1982a,b; Schönwiesner et al. 2002; Yang et al. 2000). The studies employing MEG demonstrated a discrete, stepped progression of frequency sensitivity (from low to high) along the anterolateral to posteromedial axis of the transverse temporal gyrus (Heschl's gyrus; HG), the site of primary auditory cortex (AI) (Galaburda and Sanides 1980; Rademacher et al. 1993). Yang et al. (2000) reported that activation on the lateral aspect of HG (outside of primary auditory cortex) moved progressively more laterally with increasing frequency. Schönwiesner et al. (2002), also using fMRI, obtained data consistent with frequency progressions, although they did not interpret their results as indicative of tonotopy (see DISCUSSION).

Two of the studies using multiple stimulus frequencies have provided evidence for as many as two distinct tonotopic progressions in the human auditory cortex (Cansino et al. 1994; Pantev et al. 1995). Both observed a progression on HG, in addition to a second discrete progression of frequency sensitivity. The second mapping reported by Cansino et al. (1994) was along the inferior and lateral aspect of the superior temporal gyrus. The additional mapping reported by Pantev et al. (1995) was on the lateral aspect of HG and exhibited a mirrorimage organization of the progression located on the superior aspect of the gyrus.

Note that the discrete samplings performed in these functional neuroimaging studies are insufficient to demonstrate the presence of a tonotopic mapping that is continuous as a function of position. The identification of areas of sensitivity to "high" and "low" frequencies does not provide information regarding the frequency sensitivity of the intervening regions of cortex, either in terms of the neuronal BF or the variation of $\mathrm{BF}$ as a function of position. However, it may be reasonably argued that the progressions demonstrated using more than two stimulus frequencies suggest the existence of an underlying tonotopic progression of neuronal $\mathrm{BF}$ as a function of position.

It is unlikely that the one or two potential tonotopic mappings observed in these previous experiments represent the entire tonotopic organization of the human auditory cortex, especially given the greater number of tonotopic maps seen in other species. Human auditory cortex has been demonstrated (via fMRI) to possess as many as eight regions that exhibit greater sensitivity to either higher or lower frequencies (frequency-dependent response regions, FDRRs) (Talavage et al. 2000). It was hypothesized that certain pairs of these higherand lower-frequency FDRRs may represent responses from different portions of the same tonotopic maps and that four or more tonotopic maps may exist in human auditory cortex. Here, we test this proposal.

In this fMRI study, we identified tonotopically organized areas within human auditory cortex using an approach inspired by techniques for mapping retinotopy in the visual system. The experimental paradigm involved slowly sweeping the center frequency of a narrow-band noise from high to low (or low to high), identifying all responsive locations in auditory cortex, and, for each such location, determining the latency of response relative to onset of the sweep. Because acoustic frequency varied systematically with time during the sweep stimulus, response latency served as an indicator of frequency sensitivity. Specifically, the frequency to which a cortical location was maximally sensitive was presumed to covary with response latency. Areas of cortex that showed a progressive change in response latency with position (and hence a progressive change in the frequency of maximal sensitivity) were considered "tonotopically organized." By mapping the data of the present study into the coordinate system (i.e., Talairach and Tournoux 1988) used by Talavage et al. (2000), we were able to compare the locations of identified tonotopic areas to those of previously defined FDRRs.

Through this work, we identified six spatial progressions of frequency sensitivity on the human superior temporal lobe. Our data, combined with previous anatomical work, lead us to suggest that the progressions correspond to six tonotopic mappings within five of the auditory areas defined on an anatomic basis by Galaburda and Sanides (1980).

\section{METHODS}

\section{Subjects}

Seven adult subjects ( 5 male, 2 female; ages 22-35) participated in 16 functional imaging sessions with each individual subject being imaged in 1-5 sessions. Each subject further participated in a separate anatomical imaging session. All subjects had normal hearing in the range from 100 to $8,000 \mathrm{~Hz}$. Informed consent was obtained from the subjects prior to each imaging session. Experimental protocols were approved by human studies committees at the Massachusetts Institute of Technology, Massachusetts Eye and Ear Infirmary and the Massachusetts General Hospital.

\section{Acoustic stimulation}

STIMULUS. Subjects were repeatedly stimulated monaurally in the right ear with a narrow bandwidth, amplitude-modulated noise that was swept in center frequency. Specifically, the center frequency was swept exponentially over the course of $64 \mathrm{~s}$ between end frequencies of 125 and $8,000 \mathrm{~Hz}$. A bandwidth proportional to the center frequency ( 0.3 octaves) was used because an approximately logarithmic variation in $\mathrm{BF}$ with linear distance has been observed in the cortex of many species (e.g., Merzenich et al. 1975), and we desired to excite regions of comparable extent for each center frequency. AM was at 8 $\mathrm{Hz}$ with a modulation index of $0.7 .{ }^{1}$ Eight sweeps were presented in each functional imaging "run" with no delay between sweep presentations. Within each run, the center frequency in all eight sweeps was directed UP from 125 to $8,000 \mathrm{~Hz}$ or DOwN from 8,000 to $125 \mathrm{~Hz}$. Between three and eight runs of a particular sweep were performed in each imaging session. Results focus on the six subjects (1-6) for whom both UP and DOwN sweeps were used. The number of UP and DOWN sweep runs conducted and analyzed for each subject is indicated in Table 1.

STIMULUS LEVEL. Acoustic stimulation levels were 35 (9 sessions) or $40 \mathrm{~dB}$ (7 sessions) above behavioral hearing threshold. To account

\footnotetext{
${ }^{1}$ Amplitude modulations were used to enhance the responses from auditory cortex (Schreiner and Urbas 1988).
} 
TABLE 1. Number of DOWN and UP sweep runs conducted on each subject ( 8 sweep presentations per run)

\begin{tabular}{ccc}
\hline \hline Subject & Down Sweep Runs & Up Sweep Runs \\
\hline 1 & 24 & 3 \\
2 & 35 & 8 \\
3 & 25 & 7 \\
4 & 34 & 10 \\
5 & 14 & 4 \\
6 & 10 & 7 \\
\hline
\end{tabular}

for any masking due to the imaging noise ${ }^{2}$ thresholds were measured in the MR imager under the same acoustic conditions as during functional imaging. Hearing thresholds were obtained for 0.3 octave bandwidth noise bursts (50-ms duration) for eight center-frequencies (100, 200, 500, 1000 2000, 4000, 8,000, and 10,000 Hz) using a standard up-down method. The sweeps used during functional imaging were digitally generated for each subject such that intensity was a function of the center-frequency, following that individual's interpolated (log-linear) threshold curve. The stimulation levels used ${ }^{3}$ were chosen in an attempt to limit the activation of the "tails" of the tuning curves and thus restrict upward spread of activation in the cochlea. Nevertheless, given that the stimulation levels were not at threshold, we anticipate cortical neurons responded to frequencies somewhat below, as well as at, BF, in accordance with the low-frequency bias of frequency tuning curves.

STIMULUS PRESENTATION. All stimuli were played through LabVIEW on a Macintosh Quadra computer outfitted with a D/A board (National Instruments A2100), and presented via the pneumatic delivery system described in Talavage et al. (2000).

TASK. In all sessions, subject attention to the stimulus was maintained by an intensity change detection task. Subjects were requested to attend to the stimulus and to raise their right index finger when they detected small $( \pm 3 \mathrm{~dB})$ intensity changes of 2-s duration that were incorporated into the sweep at random intervals (5-10 per 64-s sweep). These intensity changes were often difficult to detect because the intensity of the stimulus followed the subject's interpolated audiogram as described in the preceding text. Subject performance was monitored by the experimenter. A run was discarded if the subject failed to detect $\geq 50 \%$ of the intensity changes.

\section{Anatomical imaging sessions}

For each subject, an anatomical imaging session was conducted to obtain the volumetric data required for cortical surface reconstruction and flattening (Dale and Sereno 1993; Dale et al. 1999; Fischl et al. 1999). High-resolution $(1 \times 1 \times 1.2 \mathrm{~mm})$ T1-weighted "reference" anatomical images with high gray matter-white matter contrast were acquired for the entire brain (124 slices). The images were obtained for five subjects on a $1.5 \mathrm{~T}$ Siemens (Erlangen, Germany) Vision imager and for two subjects on a 1.5 T General Electric (Milwaukee, WI) Signa imager.

\section{Functional imaging sessions}

All functional images were acquired on a General Electric $1.5 \mathrm{~T}$ Signa imager, equipped for echo-planar imaging by ANMR (Wil-

\footnotetext{
2 The imaging noise reached a maximum level of $115 \mathrm{~dB}$ SPL at $1 \mathrm{kHz}$ with harmonics $\leq 8 \mathrm{kHz}$ exhibiting sound levels $>70 \mathrm{~dB}$ SPL (Ravicz et al. 2000). The earmuffs of the sound presentation system attenuated the noise by 30-35 $\mathrm{dB}$ (Ravicz and Melcher 2001), resulting in noise levels at the ear of 80-85 dB SPL.

${ }^{3}$ Based on measurements of subject thresholds both in silence and in the presence of acoustic imaging noise, and measures of hearing threshold levels for humans (e.g., Fig. 6.3 of Fletcher 1992), absolute presentation levels are estimated to have been in the range $40-80 \mathrm{~dB}$ SPL.
}

mington, MA). For functional imaging, an asymmetric spin-echo sequence $(\mathrm{TE}=70 \mathrm{~ms}, \tau=-25 \mathrm{~ms}, 128 \times 64$ acquisition matrix, in-plane resolution $=3.1 \times 3.1 \mathrm{~mm}, \mathrm{TR}=2 \mathrm{~s})$ was used to acquire 256 images of each of eight (14 sessions) six (1 session), or five (2 sessions) axial oblique slices (3-mm thick with a 1-mm inter-slice gap). The imaged slices were selected to encompass HG and much of the superior temporal plane. Immediately prior to functional imaging, T1-weighted anatomical images were obtained of the functionally imaged slices. These "single-session" anatomical images were used to register the functional data from that session to the "reference" anatomical images. Both the single-session anatomical images and the functional images were acquired using a unilateral, receive-only surface coil positioned over the left hemisphere (Talavage et al. 2000). ${ }^{4}$ Subject motion was limited in all sessions through use of a dental bite bar made prior to each imaging session.

\section{Data processing}

CORTICAL FLATTENING. For each subject, the high-resolution reference anatomical images were used to generate a patch of flattened cortex containing much of the superior temporal plane, including HG, Heschl's sulcus (HS), and the superior temporal gyrus (STG). This flattened format enabled the identification of continuous variations of frequency sensitivity across the cortical surface that would be obscured in a slice-by-slice analysis of the data. The flattened cortex was generated using the procedure of Dale and Sereno (1993) and is outlined here. First, the high contrast between gray matter and white matter in the reference anatomical images permitted the automated detection of the outline of the white matter in each slice. The slices were combined into a single three-dimensional volume, and the surface of the white matter was constructed by "shrink-wrapping" the brain. This surface (Fig. 1a) was then inflated, with minimized distortion, to eliminate the folding of the gyri and sulci (Fig. 1b). Next, a patch enclosing the superior temporal plane (black outline in Fig. $1 b)$ was computationally "cut" from the three-dimensional representation of the cortical surface. The remaining curvature of this patch was then removed, computationally, to yield a flat patch of auditory cortex (Fig. 1c).

The flat patch provides a convenient opportunity to examine variation of activity across the three-dimensional surface of the cortex in a single view. Approximate indications of direction in three-dimensional space have been provided in Fig. $1 c$ to orient the reader who is familiar with the anatomy of the superior surface of the temporal lobe with the flat patch representation of this same anatomy. However, these approximate directions should not be interpreted as axes-linear movement on the flat patch does not equate to linear movement within the three-dimensional volume of the brain. Rather, linear movement across the surface of the patch corresponds to complex movement along the folded surface of the cerebrum. For example, movement along HG from image left to image right in Fig. 1c (approximately posteromedial) actually comprises a superior movement from the anterolateral junction with the STG, a posteromedial and somewhat inferiorly directed trajectory, and an inferior movement down the medial face of the gyrus.

Correspondences between functional activity and underlying anatomical areas will be assessed using the anatomical landmarks in the flat patch, but orientation of potential tonotopic mappings and group comparisons will be evaluated using the stereotactic coordinate system of Talairach and Tournoux (1988). This operational distinction is based on Rademacher et al. (1993), in which anatomical landmarks were observed to be useful indicators of the location of particular

\footnotetext{
${ }^{4}$ The coil ( $\sim 5$-in radius) results in a visible drop-off of signal within the images, but the measured SNR remains well above the level of a standard transmit-receive head coil even at the midline. Therefore although we expect improved detection of subtle responses at the lateral aspect of the brain, we also expect meaningful detections in the medial portion of the auditory cortex and the insula.
} 
anatomical areas in auditory cortex (especially Brodmann area 41), but that the location and extent of these areas were uncertain when evaluated in standardized coordinate systems.

FUNCTIONAL DATA CONVERSION TO FLATTENED CORTICAL SPACE. Prior to data analysis, all functional imaging data for a given subject were converted into the coordinate space of the flattened cortical surface described in the preceding text. First, the data from all runs of a given imaging session were aligned (SPM95) (Friston et al.
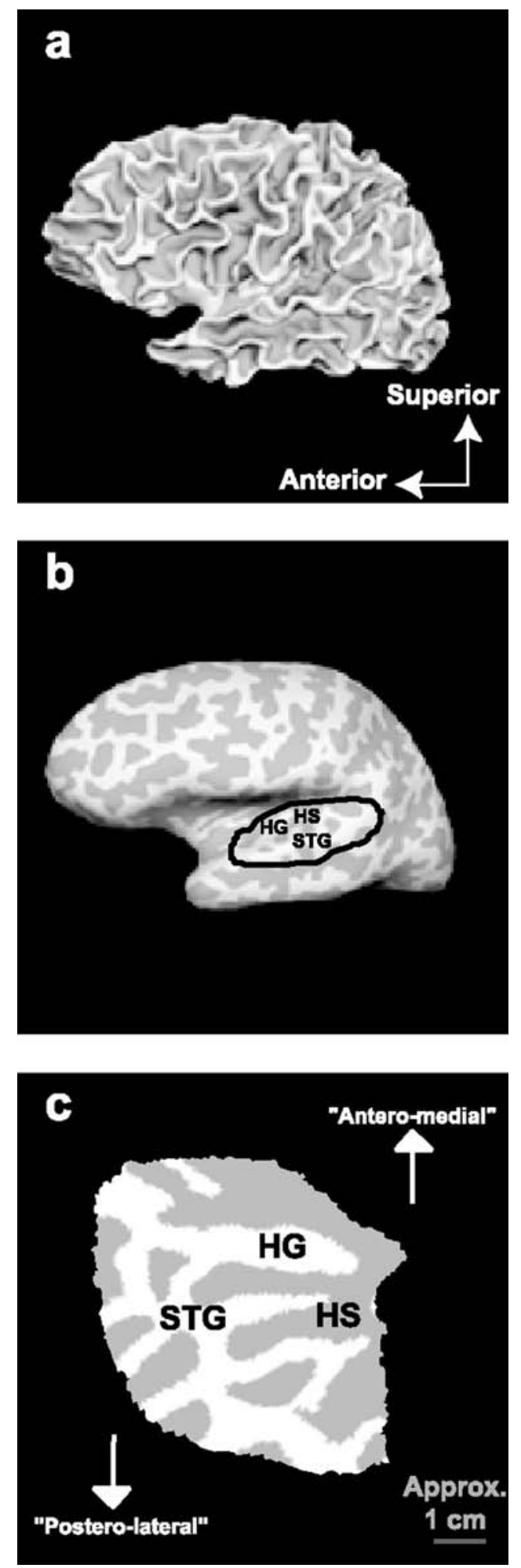

1995) to the first acquired images of the first functional run. This alignment optimizes registration of the functional data to the singlesession anatomical images. The single-session anatomical images were manually registered to the higher-resolution reference anatomical images using tools associated with FreeSurfer (Dale et al. 1999; Fischl et al. 1999), yielding an affine transformation matrix relating the two data sets. After this operation, the known relationship between the reference anatomical images and the inflated cortical surface was extended to the single-session anatomical images and, by the registration step, to the data from the functional runs. Finally, all functional data for each subject were converted to the flattened cortical space using the transformation matrix obtained for the single-session anatomical images.

FUNCTIONAL DATA ANALYSIS. After conversion to the flattened cortical space, the functional data were analyzed to identify cortical locations responsive to the sweep stimuli and, for each responsive location, to determine the latency of response relative to sweep onset. Although the detailed analyses were performed in the Fourier domain (see following text), the results will be presented in the time domain (i.e., in terms of response amplitude and latency).

The multiple data sets acquired for a given subject were combined in a two-step process to obtain a single data set for each subject. First, the temporal variation of the image signal for each run of UP or DOwN sweeps was analyzed using Fourier techniques to determine, for each location on the cortical surface, the signal magnitude and phase-angle at the rate of sweep presentation ( 8 per run), as per Sereno et al. (1995). The magnitude and phase-angle of the Fourier component at a given surface location comprise the "average response vector" for that location. The magnitude indicates the average amplitude of the response to the sweep. The phase angle (given as a fraction of $164-\mathrm{s}$ sweep) is proportional to the latency of response. ${ }^{5}$ Second, for all surface locations, a "total response vector" was computed by adding the conjugate of the average response vectors for UP sweeps to the average response vectors for DOwN sweeps. In effect, conjugation reverses the temporal order of the UP sweep data to permit analysis as if acquired in response to a sweep of the opposite direction (i.e., DOwN). The total number of runs combined in this fashion for each subject is shown in Table 1, ranging from 17 (subject 6; 10 Down, 7 UP) to 44 (subject 4; 34 DOWN, 10 UP).

Statistical analysis (Fourier $F$-test) of the total response vectors was used to determine the information to be displayed for each point on the inflated cortical surface. For each point, the summed energy in three-point neighborhoods centered at the rate of stimulus presentation ( 8,16 , and 32 per run) was compared with the total spectral energy. Those points on the cortical surface achieving $P<0.001$ were

\footnotetext{
${ }^{5}$ Note that in the DOwN reference frame the tails of tuning curves will result in detection of the peak response at a time subsequent to presentation of the BF for a given location (assuming that the BF roughly approximates the upper frequency limit of the tuning curve).
}

FIG. 1. Generation of a flat patch of left superior temporal cortex (subject 1) per Dale and Sereno (1993). $a$ : surface of white matter constructed from high-resolution (voxel size $=1 \times 1 \times 1.2 \mathrm{~mm}$ ) anatomical images with high gray matter-white matter contrast. $b$ : surface of white matter inflated and computationally area corrected to yield a smooth surface. Tops of gyri (defined by outward curvature) are colored light gray while sulci (defined by inward curvature) are colored a darker gray. Patch of the left superior temporal plane (black outline) is selected to contain Heschl's gyrus (HG), Heschl's sulcus (HS), and the superior temporal gyrus (STG). $c$ : the outlined patch in $b$ is detached from the inflated surface, further flattened, and rotated to more closely resemble an axial image of the left superior temporal plane. Approximate directions have been indicated to orient the reader to the superior surface of the temporal lobe. It is important to note, however, that orthogonal axes no longer apply after the inflation and flattening process as linear motion in the 3-dimensional volume of the brain does not correspond to a linear trajectory on the flat patch. Rather linear trajectories on the patch correspond to complex movement along the surface of the cerebrum. 


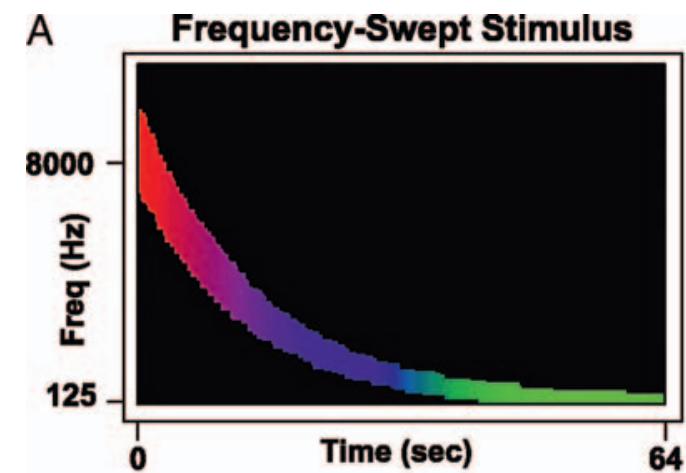

B

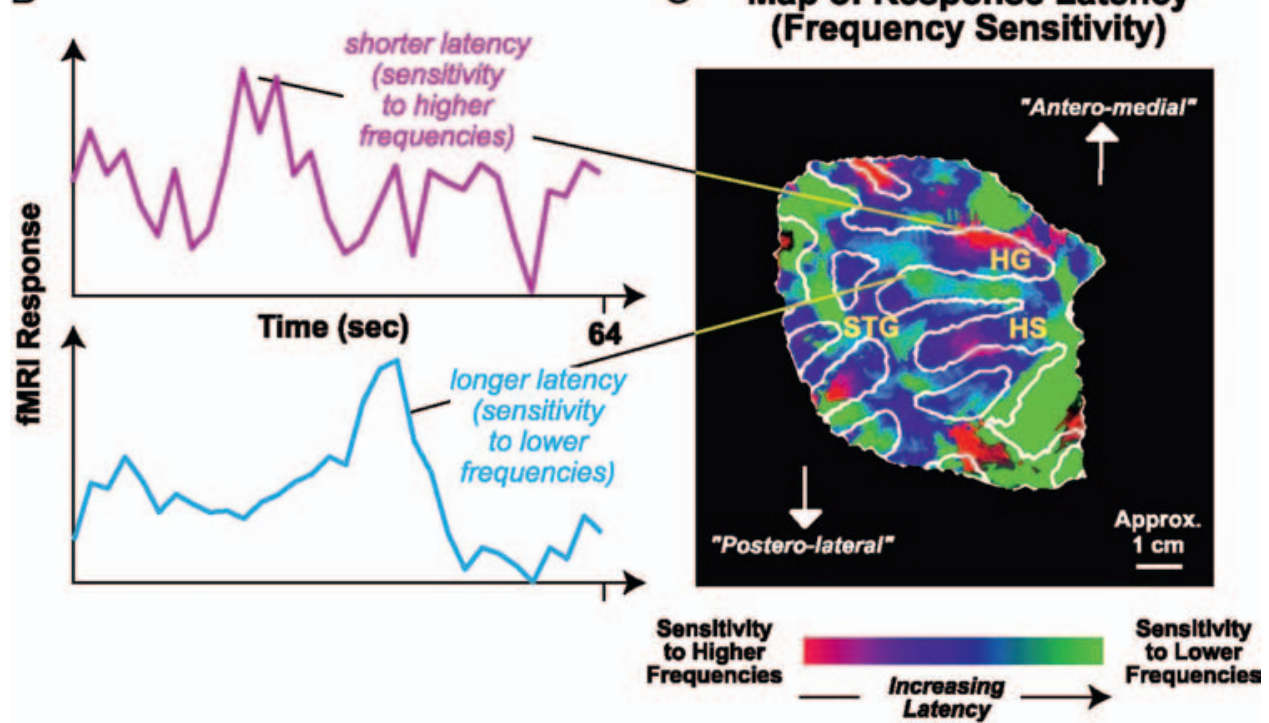

FIG. 2. Relationship between frequencyswept stimulus, functional magnetic resonance imaging (fMRI) response, and cortical frequency sensitivity. $A$ : stimulus frequency composition vs. time. $B$ : actual fMRI responses from 1 session (average of 8 DOWN runs) for subject 1 for 2 cortical locations. The first (pink) shows a shorter latency response and hence sensitivity to the higher frequencies occurring early during the stimulus sweep. The second (cyan) shows a longer latency response indicating sensitivity to lower frequencies occurring later in the sweep. $C$ : color map of response latency displayed on the flattened patch of cortex from Fig. 1c (subject 1). This map can alternatively be viewed as a map of frequency sensitivity. Red/magenta and cyan/green indicate sensitivity to higher and lower frequencies, respectively. The white outline indicates the border between gyri and sulci (i.e., the light and dark gray in Fig. 1c). then displayed with intensity proportional to the $P$ value and color related to the phase-angle at the rate of stimulus presentation. (Because of the proportional relationship between phase angle and latency of response, the color can alternatively be viewed as an indicator of response latency.) For locations on the surface that do not respond to the same frequency range in both the UP and DOwN sweeps, the phase angle of the session average vectors will be random and addition will yield a magnitude near zero. Therefore points on the surface not strongly selective for stimulus frequency will have minimal statistical significance.

This technique relies on the latency of the hemodynamic response to a neuronal event being largely invariant across regions of cortex. In fact, there are known region-to-region differences in this latency presumed to be due to regional differences in the coupling of the vascular system to the underlying neuronal activity. However, these latency differences are typically on the order $\leq 3 \mathrm{~s}$ (e.g., Saad et al. 2001) and therefore do not pose a problem for the present study because they are far shorter than the long duration (64 s) of the stimulus sweep.

The procedure described in the preceding text assumes that the relationship between stimulus frequency and response phase angle is the same for DOWN and (conjugated) UP sweep data. However, there are factors that could prevent this from being true. Therefore we also analyzed the data for UP and DOwN sweeps separately, for comparison. There are two factors that could lead to somewhat different results for UP and DOWN sweeps. One is the delay of the blood-oxygenation-leveldependent (BOLD) fMRI response (Bandettini et al. 1993; Kwong et al. 1992; Ogawa et al. 1990, 1992). Therefore when the UP sweep is conjugated for addition to the DOwN sweep, the BOLD response delays produce some phase-angle mismatch, reducing the overall magnitude of the response and yielding a weighted-average measurement of the observed response delays. The other factor of concern is the low-frequency bias of the frequency tuning curves in the auditory periphery. ${ }^{6}$ This bias could result in a different relationship between stimulus frequency and response phase-angle for (conjugated) UP as compared with DOwn sweeps. Despite these factors, the data for UP and DOwn sweeps, when analyzed separately, exhibited the same basic trends, indicating that the combination of UP and DOWN sweep data (as in all presented results) was reasonable.

MAPPING FDRR FOCI TO FLATTENED CORTICAL PATCHES. The reference anatomical data were mapped into the Talairach coordinate system so that the average Talairach locations of FDRR "foci" of Talavage et al. (2000) could be superimposed on the flattened cortical patches. A "focus" is the most significant (i.e., lowest $P$ value) voxel within an FDRR. Only the most consistently observed FDRR foci (i.e., "numbered" FDRR foci) are considered here.

\section{RES ULTS}

\section{Identification of frequency sensitivity in response} to swept stimuli

Figure 2 depicts a map of frequency sensitivity in the flattened auditory cortex of one subject and illustrates its relation-

\footnotetext{
${ }^{6}$ Because of the low-frequency bias in auditory tuning curves, we initially thought that DOWN sweeps might provide greater frequency specificity than UP sweeps. Therefore we initially concentrated on obtaining DOWN sweep data, hence the larger number of DOWN runs compared with UP runs in each subject (Table 1).
} 


\section{Wavefronts and Relative Frequency Sensitivity in Auditory Cortex}

\section{Subject 1}
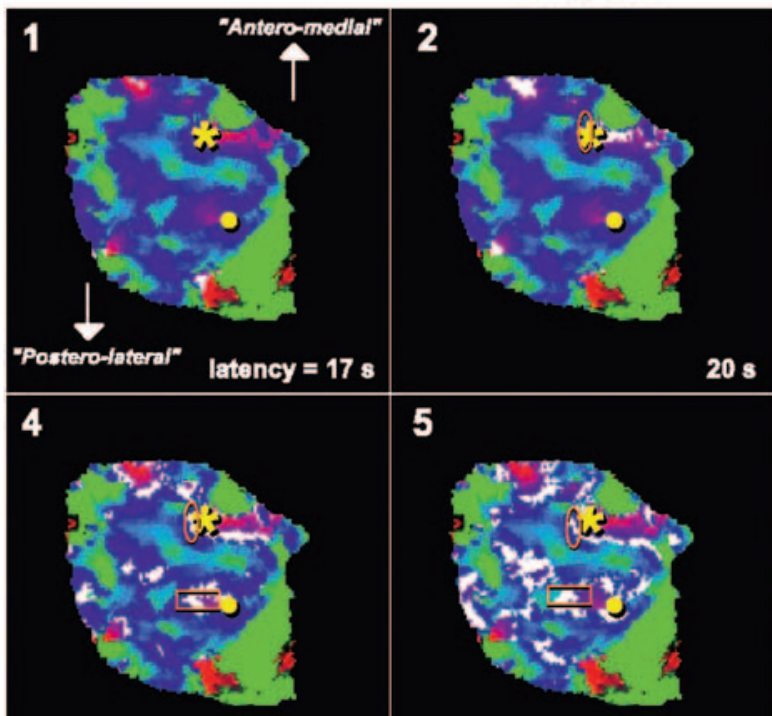

5

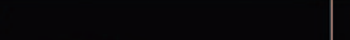

$27 \mathrm{~s}$

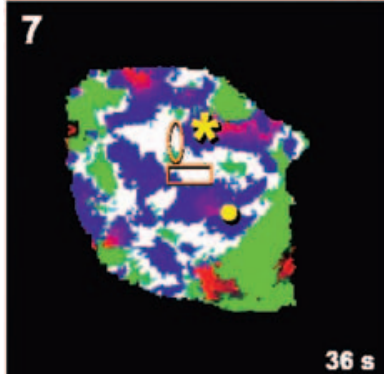

$36 \mathrm{~s}$
8

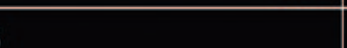

9
3

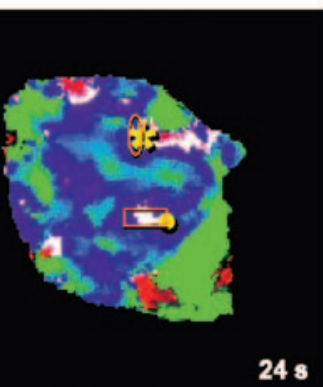

6

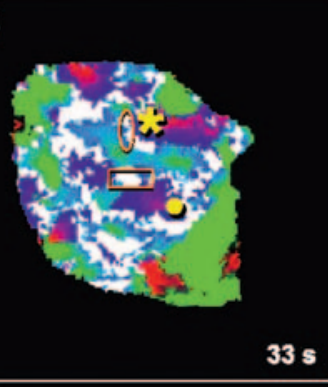

$33 \mathrm{~s}$

48

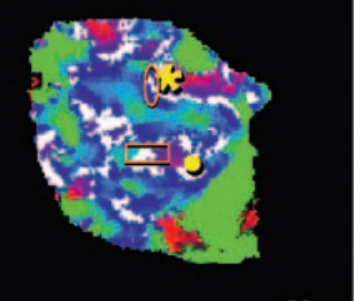

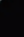

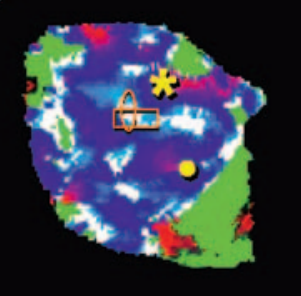

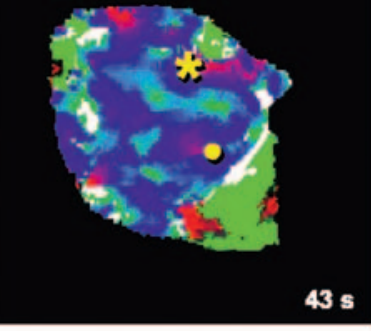

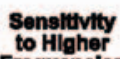

to Higher
$40 \mathrm{~s}$

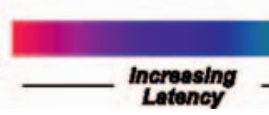

$43 \mathrm{~s}$

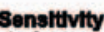

Sensilutity

Frequencles
FIG. 3. Wavefronts and relative frequency sensitivity in the left superior temporal plane of subject 1. Each panel shows a color map of latency (i.e., frequency sensitivity) displayed on the flattened patch of cortex in Fig. 1C. The superimposed wavefronts (white; contours of latency in a 6.4-s window centered on the indicated time) correspond to latencies ranging from shortest in 1 to longest in 9 . Comparing the 9 panels, multiple wavefronts can be observed progressing across the cortical surface from regions of shorter latency (red/magenta) to regions of longer latency (cyan/green). Two example wavefronts have been indicated by the oval and rectangle. One wavefront (oval) originates in 2 on the anteromedial aspect of HG (at star), moves left in the image to terminate on the anterolateral aspect of $\mathrm{HG}$ in 8 . The 2 nd wavefront (rectangle) originates in the posterior portion of HS (at dot) in 3 , moves toward the top left of the image, and terminates just posterior to $\mathrm{HG}$ in 8 , merging with the previous wavefront. Note: due to the 6.4-s window, the depicted response latencies correspond to peak responses occurring 13-47 s postonset (this interval corresponds to presented center frequencies between 3,400 and $380 \mathrm{~Hz}$ ). Several inferences may be made from these limits. First, the lower limit indicates that insignificant peak-response activity was observed prior to $13 \mathrm{~s}$. This is not surprising given that all detected latencies in the Down reference frame must necessarily lag the time of presentation of the best frequency (BF). Second, the upper limit indicates that few locations exhibited peaks centered at latencies $>47$ s postonset. This suggests that minimal new activity arose in the cortex once the stimulus content had dropped $<500 \mathrm{~Hz}$. This is also not surprising as significant phase-locking in the cochlea would likely produce some level of activity throughout the cortex (independent of BF) once the stimulus content dropped $<1 \mathrm{kHz}$ (32 s postonset). ship to a frequency-swept stimulus. As stimulus center frequency was swept (from high to low in Fig. $2 A$ ), different locations in auditory cortex responded at different latencies (Fig. 2B). Because acoustic frequency varied systematically with time during the sweep, response latency was directly related to frequency sensitivity. Specifically, locations responding at shorter latencies were presumed to be sensitive to higher frequencies, whereas locations responding at longer latencies were presumed to be sensitive to lower frequencies. It is important to recognize that the approach of the present study precludes the assignment of absolute frequency sensitivity (e.g., $1 \mathrm{kHz}$ ) to any portion of cortex. Instead, it provides information concerning the relative frequency sensitivity of different cortical locations.

Figure $2 C$ shows response latency spatially mapped on the flattened cortex of one subject (subject 1). For all cortical locations showing a significant response to the sweep stimuli $(P<0.001, F$-test $)$, response latency is indicated using a continuous color gradient such that locations with the shortest latencies are red/magenta, those with intermediate latencies are blue, and those with the longest latencies are cyan/green. The map of Fig. $2 C$ can alternatively be viewed as a map of frequency sensitivity with areas sensitive to higher frequencies corresponding to sites of shorter latency responses (red/magenta) and areas sensitive to lower frequencies corresponding to sites of longer latency responses (cyan/green). ${ }^{7}$

Further appreciation for the data underlying maps of frequency sensitivity can be obtained by tracking response "wavefronts" along the cortical surface. A wavefront consists of voxels (with $P<0.001, F$-test) exhibiting similar response latencies. ${ }^{8}$ Figure 3 (1-9) displays, on the flattened cortex of subject 1, the wavefronts (in white) for nine sequential latencies superimposed on a color map of relative frequency sensitivity for the same subject. The wavefronts corresponding to the shortest latencies ( 1 and 2 ) are localized to regions sensitive to higher frequencies (red/magenta in the map), while the wavefronts corresponding to the longest latencies ( 8 and 9 ) are localized to regions sensitive to lower frequencies (cyan/

\footnotetext{
7 The frequency-sensitivity maps presented are based on UP as well as DOWN sweeps. However, because the UP sweep data were converted to the DOWN sweep reference frame (see METHODS), shorter response latencies always indicate sensitivity to higher frequencies, whereas longer latencies indicate sensitivity to lower frequencies.

${ }^{8}$ For all analyses, wavefronts were constructed using cortical locations exhibiting peak response during a 6.4-s window centered on the current latency.
} 
green). Between these two latency extremes, there are progressive changes in wavefront position with latency, as can be seen by tracking two of the wavefronts in Fig. 3 (indicated by oval and rectangle) as they move away from their position at short latencies (indicated by star and dot, respectively). One wavefront (oval) progresses primarily to image left with increasing latency (3-7) until it merges with other wavefronts (8). The second wavefront (rectangle) progresses toward the top left (4-8) with increasing latency. These two progressions approximate anterolateral (oval) and anterior (rectangle) displacement as a function of time. These progressions in wavefront position, indicating spatial progressions in frequency sensitivity, are captured in the color map as a continuous change from red/ magenta, to blue, to cyan/green. We interpret each progression as a single tonotopic gradient.

\section{Progressions of frequency sensitivity}

Progressions of frequency sensitivity that occurred repeatably across subjects were identified as follows. Initially, for each subject, all sites of highest- and lowest-frequency sensitivity were identified (i.e., wavefront locations at the shortest and longest latencies were identified). Then the "connectivity" between these sites was determined by tracking wavefronts along the cortical surface (as in Fig. 3). In this way, spatial progressions in frequency sensitivity were identified for each subject. Comparisons were then made to identify progressions that repeatedly occurred across subjects. These comparisons looked for progressions that repeatedly occurred in conjunction with a particular anatomical landmark (e.g., the anteromedial aspect of $\mathrm{HG}$ ) or at a particular location relative to other progressions. The location of endpoints, relative to anatomical landmarks, were determined using the flat patch, whereas the orientation of the primary axis of a progression was assessed using Talairach coordinates (see Rademacher et al. 1993). For progressions that occurred in at least half of the six subjects, the higher- and lower-frequency "endpoints" of the progression were designated with primed numbers [corresponding to the closest "numbered" FDRR of Talavage et al. (2000)] and are described in the following text. All other progression endpoints were left undesignated and were not analyzed further. In the end, six frequency progressions were identified which linked seven labeled endpoints.

Three progressions of frequency sensitivity were observed in consistent locations on and near HG in all six subjects. One progression connected a region of higher-frequency sensitivity anteromedially on HG (endpoint 2', red in Fig. 4) with a region of lower-frequency sensitivity directly on HG (endpoint 1', green). A second progression was observed near HG, connecting endpoint $3^{\prime}$, a higher-frequency endpoint (red or magenta for subjects $1-4$ and 6 ; purple for subject 5) in HS posterior to HG (HG-2 in cases with a bifurcated HG), with the lowerfrequency endpoint $1^{\prime}$. The third progression observed in every subject also originated at the higher-frequency endpoint $3^{\prime}$ and terminated at endpoint $6^{\prime}$ in a region of lower-frequency sensitivity located on the STG, posterolateral to the main body of HG.

Three further progressions of frequency sensitivity were observed in the superior temporal plane (STP; that portion of the temporal lobe medial to and including the STG) in at least four of the six subjects. The most common of these progres- sions, observed in five subjects, originated in the region of higher-frequency sensitivity corresponding to endpoint $3^{\prime}$ and terminated in the (usually large) region of lower-frequency sensitivity in the posterior STP, corresponding to endpoint $8^{\prime}$ (subjects 1, 2, and 4-6). The two remaining progressions were both observed in four of the subjects. One originated in the region of higher-frequency sensitivity containing endpoint $2^{\prime}$ and ran anterolaterally along $\mathrm{HG}$ to terminate at endpoint $7^{\prime}$ in a region of lower-frequency sensitivity on the superior aspect of the STG, in the anterior STP (subjects 3-6). Another progression originated in a region of higher-frequency sensitivity at the lateral edge of $\mathrm{HG}$ on the STG (endpoint 5') and progressed medially and sometimes posteriorly to terminate at lower-frequency endpoint 6' (subjects 1-4).

\section{Comparison of progression endpoints and FDRR foci}

A good correspondence is observed between the endpoints of frequency progressions and the previously reported FDRR foci. The average Talairach locations of the frequency progression endpoints are listed in Table 2. In Fig. 5, these locations are compared on the flattened cortical surface with the average Talairach locations of FDRR foci from Talavage et al. (2000). In general, the locations of the FDRR foci were close to endpoints of corresponding frequency sensitivity. Progression endpoints $3^{\prime}$ and $5^{\prime}$ had average Talairach locations within approximately one voxel of the corresponding FDRR foci. (An average of only $1.8 \mathrm{~mm}$ separated endpoint $3^{\prime}$ and the focus of FDRR 3, whereas the average distance between endpoint 5' and the focus FDRR 5 was $3.8 \mathrm{~mm}$.) Two additional progression endpoints $\left(6^{\prime}\right.$ and $\left.1^{\prime}\right)$ were within two voxels of their corresponding FDRR foci. (The average location of endpoint $6^{\prime}$ was $6.0 \mathrm{~mm}$ from the average location of the focus of FDRR 6 , and endpoint $1^{\prime}$ was an average distance of $6.2 \mathrm{~mm}$ from the focus of FDRR 1.) Endpoint $2^{\prime}$ was located within three voxels $(8.7 \mathrm{~mm})$ of the focus of FDRR 2. The average locations of endpoints $7^{\prime}$ and $8^{\prime}$ were 11.0 and $13.7 \mathrm{~mm}$ distant, respectively, from the foci of FDRRs 7 and 8. Using the variation in each coordinate direction to compute a standard error (SE) distance, none of the seven endpoints was found to be significantly different in average Talairach location $(P<0.05$ level $)$ from its corresponding FDRR focus. FDRR 4 (higher frequency; not shown in Fig. 4) was only found to be close to a region of higher-frequency sensitivity in three subjects, and no progression endpoints were observed in the vicinity of the FDRR focus.

Two of the subjects participating in the present study also participated in the study of Talavage et al. (2000), so withinsubject comparisons of FDRR foci and progression endpoints can be made. In both subjects, the FDRR foci were within one standard error of the average FDRR foci locations, and within two SE measurements from the higher- and lower-frequency endpoints of the present study.

\section{I S C U S S I O N}

Six progressions of frequency sensitivity were observed in auditory cortical areas of the superior temporal lobe (Fig. 6). Using a prolonged, frequency-swept stimulus, each progression was identified as a systematic shift in fMRI response latency with position along the cortical surface. Responses 


\section{Relative Frequency Sensitivity in Human Auditory Cortex}
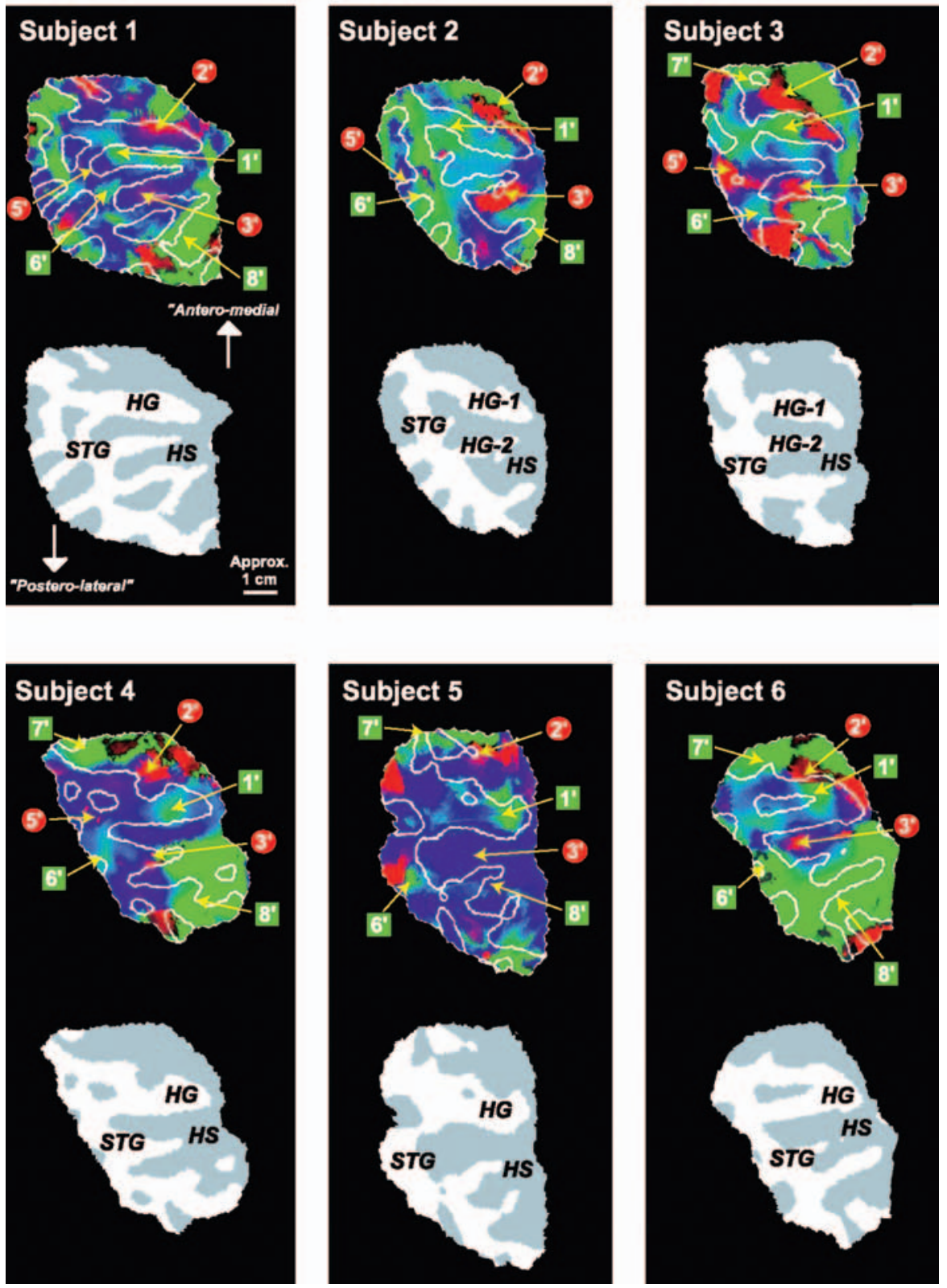

FIG. 4. Relative frequency sensitivity and anatomy of the left superior temporal plane for subjects 1-6. Top in each panel: color map of latency (i.e., frequency sensitivity) displayed on a flattened patch of cortex (same color code as Fig. 2). Primed numbers indicate the high-frequency (red circles) and low-frequency (green squares) endpoints of progressions in frequency sensitivity. Bottom in each panel: the same cortical patch with gyri and sulci shown in light and dark gray, respectively. In subjects 2 and 3, the intermediate transverse sulcus divides $\mathrm{HG}$ into the 1 st (HG-1) and 2nd (HG-2) HG. observed with fMRI as changes in image signal levels reflect changes in underlying neural activity (Kwong et al. 1992; Ogawa et al. 1990, 1992). Thus each progression identified

TABLE 2. Average Talairach coordinates and SE values of frequency progression endpoints

\begin{tabular}{cccr}
\hline \hline & \multicolumn{3}{c}{ Average Talairach Coordinates } \\
\cline { 2 - 4 } Endpoint & $x$ & \multicolumn{1}{c}{$y$} & \multicolumn{1}{c}{$z$} \\
\hline $1^{\prime}$ & $-46.2 \pm 1.4$ & $-13.9 \pm 2.7$ & $8.7 \pm 1.7$ \\
$2^{\prime}$ & $-41.7 \pm 1.9$ & $-16.8 \pm 1.5$ & $2.6 \pm 2.0$ \\
$3^{\prime}$ & $-52.1 \pm 1.4$ & $-23.7 \pm 2.6$ & $9.4 \pm 2.3$ \\
$5^{\prime}$ & $-59.8 \pm 1.7$ & $-13.5 \pm 1.9$ & $4.2 \pm 1.7$ \\
$6^{\prime}$ & $-62.7 \pm 1.1$ & $-18.0 \pm 2.9$ & $6.9 \pm 2.3$ \\
$7^{\prime}$ & $-47.5 \pm 1.3$ & $0.5 \pm 2.4$ & $-7.3 \pm 1.3$ \\
$8^{\prime}$ & $-51.5 \pm 2.1$ & $-34.6 \pm 3.5$ & $20.9 \pm 2.7$ \\
\hline
\end{tabular}

here with fMRI likely reflects a progressive change in neuronal frequency sensitivity with position along the cortical surface-a tonotopic map.

The frequency progressions interconnected three regions of higher-frequency sensitivity (endpoints $2^{\prime}, 3^{\prime}$ and $4^{\prime}$ ) and four regions of lower-frequency sensitivity (endpoints $1^{\prime}, 6^{\prime}, 7^{\prime}$ and $\left.8^{\prime}\right)$. Most of these regions, or endpoints, were located quite near a FDRR focus of comparable frequency sensitivity (endpoints 1', 3', 5', 6'; FDRRs 1, 3, 5, 6 respectively). A few were located somewhat farther from their apparently corresponding FDRR focus (endpoints 2', 7', 8'; FDRRs 2, 7, 8). This disparity in location may be partially attributable to intersubject variability because the present study and the previous FDRR study did not examine exactly the same individuals. Alternatively, the disparities may reflect the differences in stimulus between the present and previous studies (e.g., if the 


\section{Comparison of Progression Endpoints and FDRR Foci}
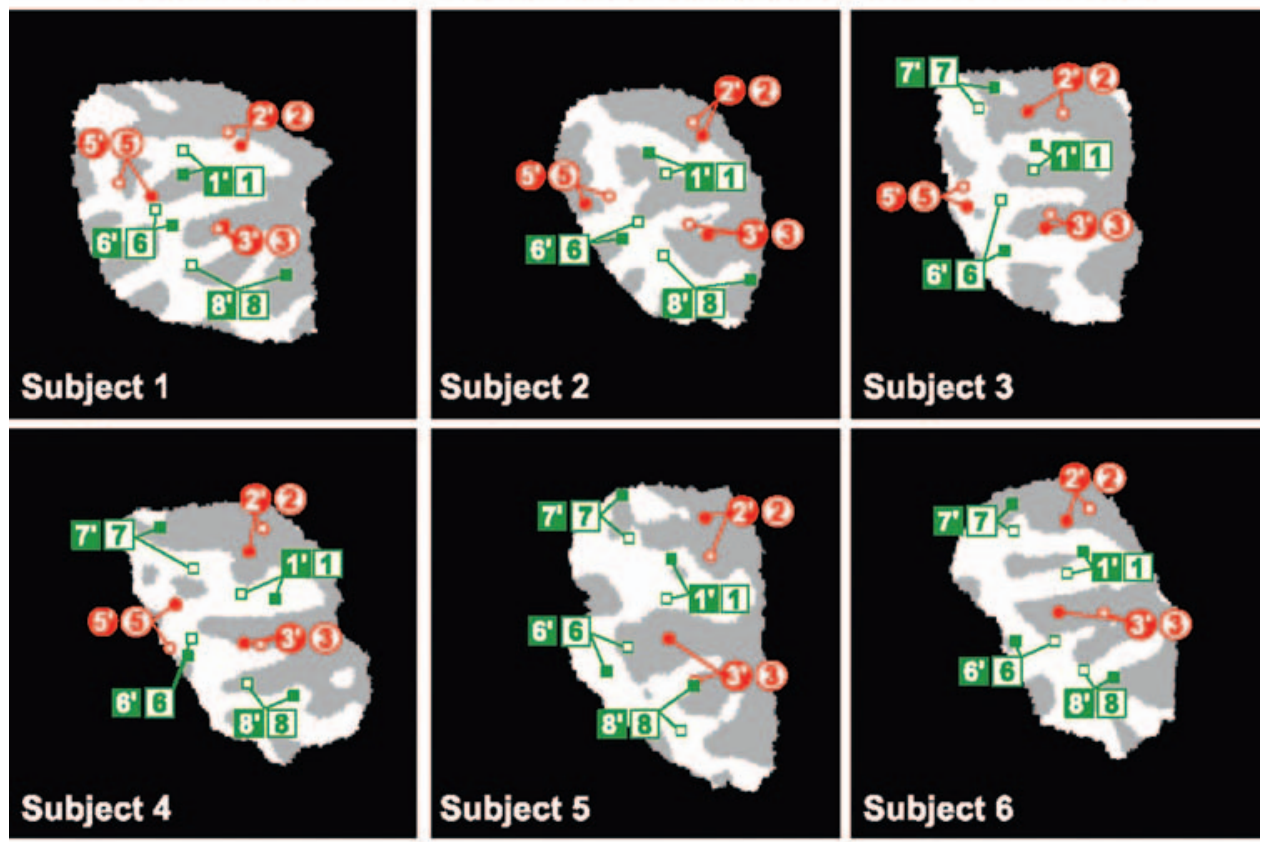

\section{Subject 2}

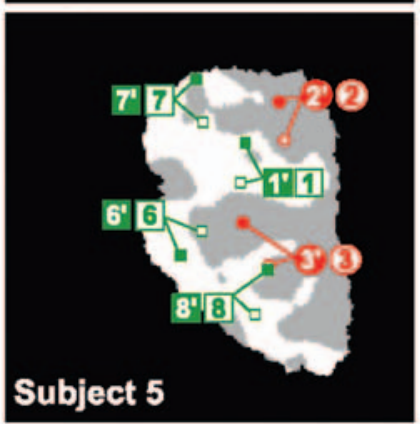

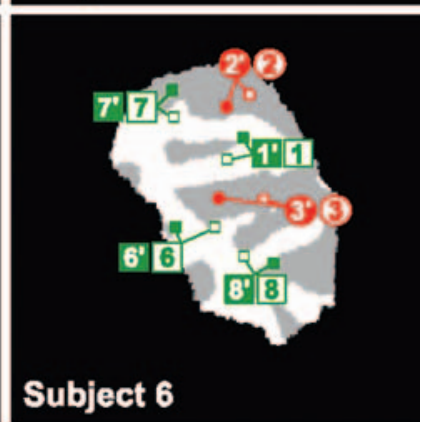

Higher Lower
Freq. Freq.

- Progression Endpoints

\section{FDRR Foci}

FIG. 5. Progression endpoints and frequency-dependent response region (FDRR) foci displayed on flattened patches of auditory cortex for 6 subjects. Closed symbols indicate the progression endpoints $\left(1^{\prime}-3^{\prime}\right.$, $\left.5^{\prime}-8^{\prime}\right)$ for each subject. Open symbols indicate the average location of FDRR foci (1-3, 5-8) from Talavage et al. (2000).

\section{Frequency Progressions in Human Auditory Cortex}

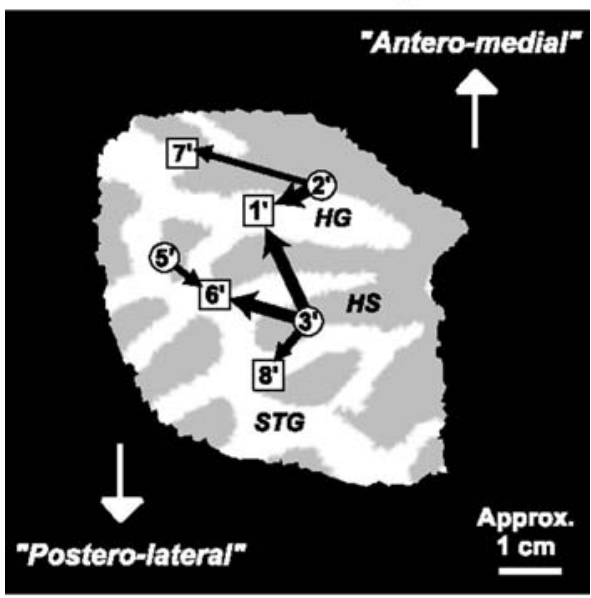

Progression of Frequency Sensitivity

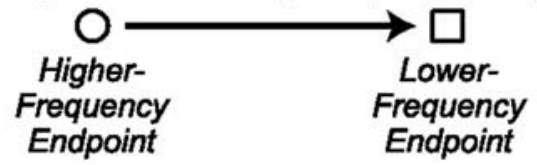

FIG. 6. Summary of 6 frequency progressions in human auditory cortex. Arrows connect endpoints between which progressions were identified in all 6 subjects (thick arrows) or in at least 4 of 6 subjects (thin arrows). The underlying cortical patch is that of subject 1. The location of progression endpoints $\left(1^{\prime}-3^{\prime}, 5^{\prime}-8^{\prime}\right)$ is qualitatively consistent with the complete set of 6 subjects. tonotopic organization of some auditory areas is revealed differently for different acoustic stimuli). Despite the methodological differences, however, both the FDRR and progression data indicate multiple (and largely the same) tonotopically organized areas.

Proposed correspondence between tonotopic maps and anatomical areas

The present study proposes a correspondence between frequency progressions and FDRRs, whereas Talavage et al. (2000) proposed a correspondence between FDRRs and anatomically distinct areas of auditory cortex (Table 3). By combining these correspondences on the basis of similarities in the spatial distribution of endpoints and FDRRs, we will now formulate hypotheses concerning the tonotopic organization of different anatomical areas within human auditory cortex. To facilitate comparison of anatomical areas, FDRRs, and frequency progressions, all three types of data are displayed in Fig. 7 in the same view as presented in Talavage et al. (2000). It is important to note that the underlying image of the brain in Fig. 7 is a single Talairach axial slice through the superior temporal lobe and that all FDRRs and endpoints have been projected onto this plane. The locations and extents of auditory cortical areas on this slice are approximate as such information is not well represented in standardized coordinate systems (Rademacher et al. 1993). Further, because the anatomy of the auditory cortex is not aligned with the orthogonal axes of Talairach space, the depicted relationship of FDRRs and endpoints to the underlying anatomy on the flat patches (Figs. 3-6) is not strictly preserved in Fig. 7. Therefore we will use the flat 
TABLE 3. Relation of frequency progression endpoints to FDRRs and anatomical auditory areas

\begin{tabular}{|c|c|c|c|}
\hline \multicolumn{2}{|c|}{ Anatomical Auditory Area } & \multirow[b]{2}{*}{ FDRRs } & \multirow[b]{2}{*}{$\begin{array}{l}\text { Corresponding } \\
\text { Endpoints }\end{array}$} \\
\hline $\begin{array}{c}\text { Galaburda \& Sanides } \\
\text { (1980) }\end{array}$ & $\begin{array}{l}\text { Rivier \& Clarke } \\
\text { (1997) }\end{array}$ & & \\
\hline KAm & AI (medial) & 2,1 & $2^{\prime}, 1^{\prime}$ \\
\hline KAlt & AI (lateral) & 3,1 & $3^{\prime}, 1^{\prime}$ \\
\hline ProA & MA & 2 & $2^{\prime}$ \\
\hline $\mathrm{PaAc} / \mathrm{d}$ & PA & 4 & - \\
\hline PaAi (anterior) & (none) & 5,6 & $5^{\prime}, 6^{\prime}$ \\
\hline $\mathrm{PaAi}$ (posterior) & LA & 3,8 & $3^{\prime}, 8^{\prime}$ \\
\hline $\mathrm{PaAe}$ (anterior) & STA & 5,8 & $5^{\prime}, 8^{\prime}$ \\
\hline PaAe (posterior) & (none) & 8 & $8^{\prime}$ \\
\hline $\mathrm{PaAr}$ & $\mathrm{AA}$ & 7 & $7^{\prime}$ \\
\hline
\end{tabular}

FDRR, frequency-dependent response region; KAm and KAlt, koniocortical divisions on Heschl's gyrus (HG); ProA and PaAr, areas anterior to HG, $\mathrm{PaAc} / \mathrm{d}, \mathrm{PaAi}, \mathrm{PaAe}$, areas posterior to the koniocortex; AI, primary auditory cortex; MA and AA, areas anterior to HG; PA, LA, and STA, areas posterior to the koniocortex.

patches to evaluate the location of FDRRs and endpoints relative to known anatomical landmarks, whereas all evaluations of endpoint and FDRR localization with respect to one another will be made using the associated Talairach coordinates.

To maintain consistency with the results presented in Talavage et al. (2000), our hypotheses are primarily presented in terms of the anatomical areas identified in two previous studies (Galaburda and Sanides 1980; Rivier and Clarke 1997) (Fig. 7, $A$ and $B)$, although reference is also made to the more recent work of Wallace et al. (2002). Galaburda and Sanides distinguished eight anatomical areas in auditory cortex based on cytoarchitectonic criteria, including two koniocortical divisions on HG (KAm, KAlt), two areas anterior to HG (ProA, $\mathrm{PaAr}$ ), and four areas posterior to the koniocortex (PaAi, PaAe, $\mathrm{PaAc/d}, \mathrm{Tpt}$ ). Rivier and Clarke identified six anatomical areas: one koniocortical area on HG (A1), two areas anterior to HG (MA, AA), and three areas posterior to the koniocortex (PA, LA, STA). Wallace et al. add to the framework of Rivier and Clarke with the identification of additional areas on the anterolateral aspect of HG (ALA) and in HS, lateral and posterior to AI (LP). Two important points should be considered regarding the attribution of endpoints and FDRRs to underlying anatomical areas. First, the extents of the anatomical areas in auditory cortex have not been defined within Talairach space, so the boundaries displayed throughout Fig. 7 are a best approximation, based on anatomical descriptions in the literature. Second, given the prominence of the mirror-image organization of tonotopic maps in experimental animals, those functionally identified endpoints across which mirror-image organizations are observed may provide a more accurate indication of the boundaries between anatomical areas or subdivisions of larger anatomical areas.

PROGRESSIONS OVERLAPPING HG. We propose that two frequency progressions correspond to tonotopic maps within KAm and KAlt. These two areas correspond to the medial and lateral portions of Rivier and Clarke's A1, which were observed by Wallace et al. to exhibit differential acetylcholinesterase staining. Note that the lateroposterior area (LP) observed in HS by Wallace et al. is likely contained within KAm and KAlt.

$K A m$ (medial A1). In all six subjects, a progression connected higher-frequency endpoint $2^{\prime}$ to lower-frequency endpoint $1^{\prime}$ along the antero-medial portion of $\mathrm{HG}$ (Fig. 7D). Endpoints 1' and 2' correspond to FDRRs 1 and 2, the foci of which lie at probable edges of KAm [near the borders of KAlt and ProA (MA); Fig. 7]. Therefore we propose that the progression linking endpoints $2^{\prime}$ and $1^{\prime}$ corresponds to a tonotopic map within KAm (medial A1).

KAlt (lateral A1). A second progression was observed in all six subjects to connect higher-frequency endpoint $3^{\prime}$ to lowerfrequency endpoint $1^{\prime}$ along the postero-lateral portion of $\mathrm{HG}$ (Fig. 7D). Endpoints $3^{\prime}$ and $1^{\prime}$ correspond to FDRRs 3 and 1, which lie at probable edges of KAlt (Fig. 7, $A$ and $C$ ). Further, a mirror-image organization is observed across lower-frequency endpoint $1^{\prime}$, suggesting that this endpoint marks a boundary between anatomical areas. Therefore we further propose that the progressions linking endpoints $3^{\prime}$ and $1^{\prime}$ correspond to a tonotopic map within KAlt (lateral A1).

PROGRESSIONS IN THE SUPERIOR TEMPORAL PLANE. We propose that four frequency progressions of the present study correspond to tonotopic maps in the following nonkoniocortical auditory areas of Galaburda and Sanides (1980) - the prokoniocortex (ProA), the internal parakoniocortex (PaAi), and the rostral parakoniocortex (PaAr). These areas correspond, at least in part, to the medial auditory area (MA), the lateral auditory area (LA), and the anterior auditory area (AA) of Rivier and Clarke (1997), and the anterolateral area (ALA) of Wallace et al. (2002). We further propose that one area$\mathrm{PaAi}$ - contains multiple divisions that are tonotopically organized. The reasoning behind these proposals is as follows.

ProA and PaAr (MA and $A A$ ). In four of six subjects, a progression between higher- and lower-frequency endpoints $2^{\prime}$ and $7^{\prime}$ was observed (Fig. 7D). These endpoints correspond to the foci of FDRRs 2 and 7, respectively. Talavage et al. (2000) proposed that higher-frequency FDRR 2 overlaps with ProA (MA) and that lower-frequency FDRR 7 corresponds to PaAr (AA). Therefore we propose that ProA (MA) and PaAr (AA) are tonotopically organized, perhaps sharing a single tonotopic map.

Anterior PaAi (ALA). ${ }^{9}$ A progression was observed in four subjects between endpoints $5^{\prime}$ and $6^{\prime}$ (Fig. 7D). These endpoints correspond to the foci of FDRRs 5 and 6, which may, in turn, correspond to the anterior portion of PaAi (Talavage et al. 2000) (Fig. 7, $A$ and $C$ ). The position of these endpoints lateral of the main body of $\mathrm{HG}$ suggests a possible correspondence with area ALA of Wallace et al. (2002). This localization, coupled with, the progression data, suggests an anteriorly positioned tonotopic map within PaAi (ALA).

Anterior and posterior PaAi. Endpoints $3^{\prime}$ and 6' were connected by a progression in all six subjects (Fig. 7D). These endpoints correspond to the foci of FDRRs 3 (higher-frequency) and 6 (lower-frequency), respectively (Fig. 5). FDRR 6 was previously proposed to be located in anterior PaAi, whereas FDRR 3 was proposed to be located posteriorly at the medial edge of PaAi (abutting KAlt; see Fig. 7, $A$ and $C$ ). Thus we propose a second tonotopic map within the anterior portion

\footnotetext{
${ }^{9}$ ALA is defined by Wallace et al. (2002). Anterior PaAi has no clear counterpart in the schema of Rivier and Clarke.
} 

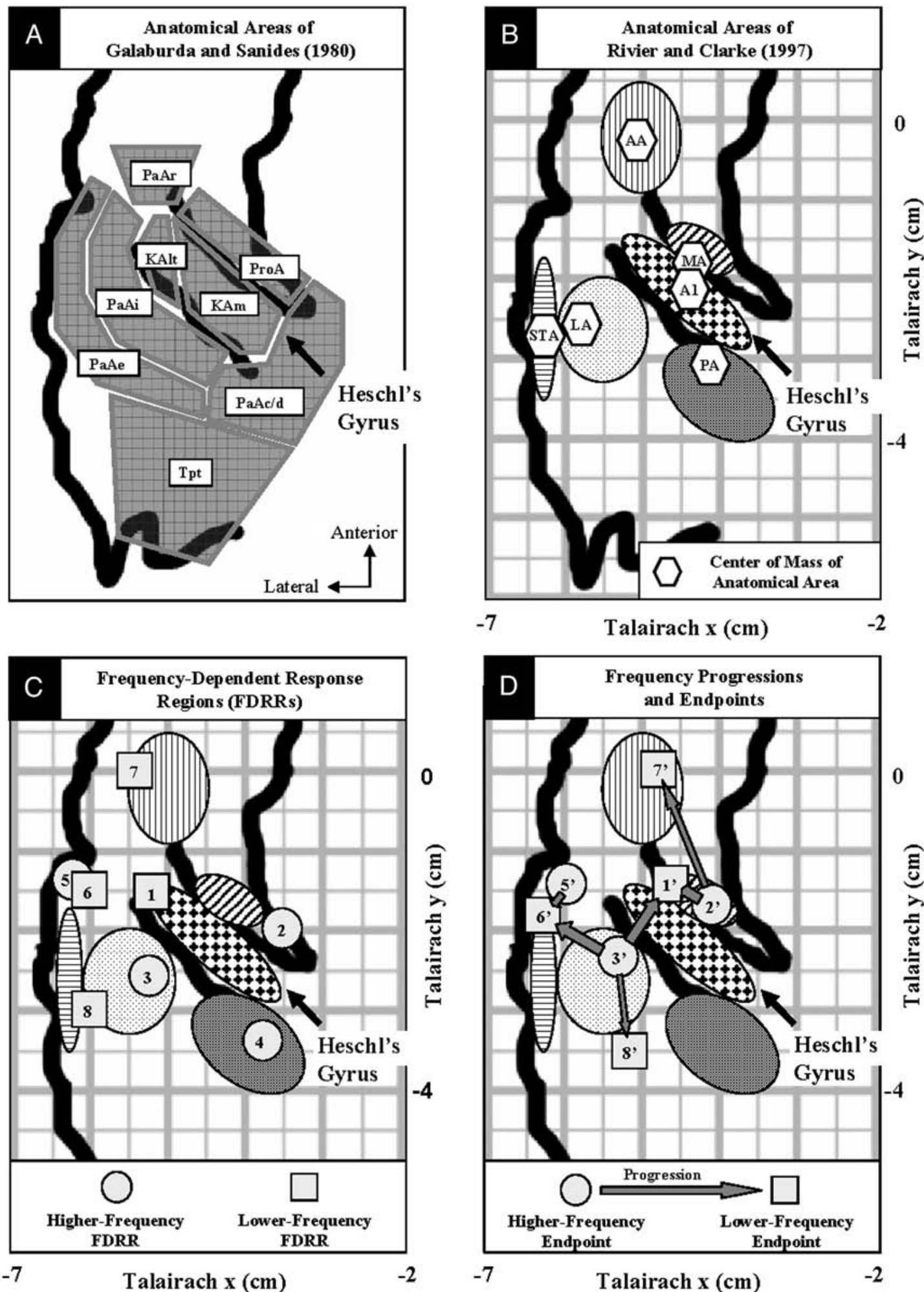

FIG. 7. Anatomical areas, FDRRs, and frequency progressions in an axial plane. Summary of anatomically defined areas from Galaburda and Sanides (1980) (A) and Rivier and Clarke (1997) (B). In B, the labeled hexagons indicate the average Talairach coordinates of the centers of mass of the anatomical areas. The patterns indicate the approximate extent of each area, but the locations of absolute boundaries in standardized coordinates are uncertain (Rademacher et al. 1993). C: average Talairach locations of FDRR foci from Talavage et al. (2000). $D$ : arrows indicate the observation, in $\geq 4$ subjects, of a frequency progression between the associated endpoints (rectangles are centered at the average Talairach locations given in Table 2). For reference, the anatomical areas of Rivier and Clarke are also shown in $C$ and $D$. The correspondence between progression endpoints and FDRR foci is as follows: (endpoint 1', FDRR 1); (2', 2); (3', 3); (5', 5); (6', 6); (7', 7); (8', 8). A-C are adapted from Talavage et al. 2000. The underlying image in all parts of the figure is based on the Talairach $z=+4 \mathrm{~mm}$ plane with the anterior and posterior extent of $\mathrm{HG}$ based on the Talairach $\mathrm{z}=+8 \mathrm{~mm}$ plane (Talairach and Tournoux 1988). 
of PaAi, exhibiting a mirror-image organization with the anterior-most map in this area, reflected across the lower-frequency endpoint 6 '.

Posterior PaAi (LA). A progression was observed to connect endpoints $3^{\prime}$ and $8^{\prime}$ in five of the six subjects (Fig. 7D), which most likely correspond to the foci of FDRRs 3 and 8, respectively. Talavage et al. (2000) assigned FDRRs 3 and 8 to the posterior portion of PaAi (LA; see Fig. 7, $A$ and $C$ ). This relationship suggests a tonotopic organization for the posterior portion of PaAi (LA). However, the correspondence of endpoint $8^{\prime}$ to the focus of FDRR 8 is the weakest of the pairings (see Fig. 7, $C$ and $D$ ), and the anatomical assignment of this progression to the posterior PaAi (LA) is thus the most tentative.

APPARENTLY NONTONOTOPICALLY ORGANIZED AREAS. The data obtained in this study do not suggest a tonotopic map within either the external parakoniocortex, PaAe (including STA) or the caudal/dorsal parakoniocortex, PaAc/d (corresponding to PA). PaAe is reported to extend from the STG onto the posterior superior temporal plane, lateral and posterior to the internal parakoniocortex (PaAi) (Galaburda and Sanides 1980; Rivier and Clarke 1997). We previously (Talavage et al. 2000) attributed two FDRRs to the anterior division of PaAe (STA)FDRRs 5 and 8 -and one FDRR (8) to the posterior division. No wavefronts were observed to connect higher-frequency endpoint 5' (corresponding to FDRR 5) with lower-frequency endpoint $8^{\prime}$ (corresponding to FDRR 8). Other than the progression connecting endpoint $8^{\prime}$ to endpoint $3^{\prime}$-attributed to the posterior division of the $\mathrm{PaAi}$ (LA) in the preceding text-no other progressions were observed to connect with endpoint $8^{\prime}$. We therefore conclude that we have not observed a tonotopic organization in either the anterior or posterior divisions of PaAe (STA). PaAc/d (PA) has been observed to cover an area posterior to the medial half of $\mathrm{HG}$ and, sometimes, to extend across the medial end of HG onto its anteromedial aspect (Galaburda and Sanides 1980). Higher-frequency FDRR 4 was previously localized to this area as a relatively robust finding of both Talavage et al. (2000) and Schönwiesner et al. (2002), but this study did not reveal a higher-frequency endpoint in the immediate vicinity of this FDRR in at least four of the six subjects. Therefore no tonotopic progression is assigned to $\mathrm{PaAc} / \mathrm{d}$ (PA).

\section{Possible discrepancies between frequency mapping techniques}

Discrepancies in proposed tonotopic maps obtained from the present study and previous work using discrete sampling techniques likely arise from differences in the mechanism by which frequency sensitivity is measured and assigned. Discrete techniques measure the relative sensitivity of cortical areas to two (or more) stimuli and attribute frequency sensitivity to that stimulus that produces the largest response. As such, discrete techniques do not provide information regarding the extent of the frequency spectrum to which the given cortical area will exhibit a nonzero response and cannot accurately predict the $\mathrm{BF}$ of the associated neurons. The technique used in this work attributes frequency sensitivity based on latency measures that primarily depend on the extent of the (presented) acoustic spectrum to which a given cortical area responds. This technique can thus provide insight regarding variation of frequency sensitivity as a function of position even though it also cannot provide an absolute measure of the sensitivity to any given frequency.

Differing assessments of frequency sensitivity are expected from the two techniques if a cortical region does not primarily consist of sharply tuned cells having BFs that vary monotonically as a function of position. In the degenerate case of an auditory cortical area that is only sensitive to a narrow portion of the acoustic frequency spectrum, the discrete techniques would lead to observation of an FDRR that would not serve as the endpoint of a progression beginning at a higher-frequency endpoint and terminating at a lower-frequency endpoint. If a cortical area possesses a tonotopic map but is largely comprised of cells exhibiting broad tuning, it is possible that neither an FDRR nor progression of a wavefront between endpoints would be observed. For example, the broad tuning could, in response to two or more stimuli, produce responses that are not significantly different and also produce latency values having such limited range that no wavefront progression could be detected.

\section{Possible dependence of progressions on stimulus level}

As noted in Talavage et al. (2000), the question of whether the pattern of cortical frequency sensitivity revealed by fMRI depends on stimulus level remains an open question. Investigations of tonotopicity are necessarily compared with work in experimental animals using microelectrode recordings. This body of literature includes results showing that the spatial pattern of activity evoked in the auditory cortex by narrowband stimuli can depend on stimulus level in complex ways (Heil et al. 1994; Phillips et al. 1994). However, it is not clear that the activation patterns observed in neuroimaging techniques such as PET and fMRI that do not exclusively rely on neuronal discharges must also exhibit complex variation as a function of level. In fact, a PET study by Lockwood et al. (1999) found little variation in response strength as a function of stimulus level. Therefore given that the activation observed with fMRI (like that of PET) reflects discharges and other synaptic activity within a given volume, the results of this work are not expected to be significantly altered by an increase or decrease in stimulation level.

\section{Is it tonotopy after all?}

Schönwiesner et al. (2002) recently reported fMRI data replicating (and adding to) the FDRRs of Talavage et al. (2000). However, they did not interpret their FDRRs as the endpoints of tonotopic maps. Instead, they attributed each FDRR to a different cytoarchitectonic area. The present results do not support this alternative view but rather support the original hypothesis of Talavage and colleagues that FDRRs are connected by frequency progressions and thus are indicative of tonotopy in human auditory cortex.

\section{Variability in the "strength" of frequency progressions}

Progressions of frequency sensitivity seen most consistently across subjects may correspond to anatomical areas that possess "stronger" tonotopic maps. By stronger tonotopic maps, we mean maps composed of cells with sharper tuning (leading to finer spatial resolution for the BOLD response), a greater 
density of tuned cells (leading to greater magnitude of metabolic demand), a lesser gradient of positional variation in $\mathrm{BF}$ (longer duration of response within a single voxel), or lower thresholds (more cells responding to the moderate intensity stimulus). For example, the progression between endpoints $2^{\prime}$ and 1', observed in all six subjects, is hypothesized to be primary auditory cortex (KAm; medial AI), an area with a high density of sharply tuned neurons that receives strongly tonotopic inputs from the ventral division of the medial geniculate nucleus (MGv) (Morel and Kaas 1992). The stronger tonotopic organization of thalamic inputs to the core regions (primarily from MGv) (Morel and Kaas 1992), coupled with the forward projections from the core to the belt, but not parabelt (Galaburda and Pandya 1983), may explain why the majority of the observed tonotopic maps may be attributed to the core and belt areas rather than parabelt $(\mathrm{PaAe})$.

\section{Imager-generated acoustic noise}

It remains an open question as to whether or not imagergenerated acoustic noise has influenced our findings. The present study attempted to reduce the effects of imager-generated noise on fMRI activation by adjusting stimulus level according to hearing sensitivity measured during functional imaging conditions. This provides compensation for threshold shifts associated with the loud imaging noise and for some of the acoustic masking. Nevertheless, we cannot rule out the possibility that the number and pattern of frequency progressions would be somewhat different under conditions of lessened interaction between the imaging noise and the stimuli [e.g., using a clustered volume acquisition with a long TR (Edmister et al. 1999; Talavage et al. 1999)].

\section{Possible interpretation of unlabeled progression endpoints}

Several endpoints at which progressions originated or terminated were not observed in three or more of our six subjects and thus remained "unlabeled." These unlabeled endpoints may reflect inter-subject differences in cortical tonotopic organization. For instance, as noted in Talavage et al. (2000) regarding the "unnumbered" FDRRs, ${ }^{10}$ the locations of some tonotopically organized areas may be sufficiently different between subjects that the repeated occurrence of a given progression and its associated endpoints went unrecognized. The differing number of UP and DOWN sweep presentations that were averaged for each subject may also contribute to the repeated observation or nonobservation of particular endpoints. While averaging data across multiple sessions improved detection of the "labeled" frequency progressions in this study, other progressions may have been more sensitive to (and thus obscured by) slight misalignments of the data from different sessions. Given the potential confounds, our identification of six tonotopic progressions potentially under-represents the number of repeatable tonotopic maps present in human auditory cortex.

\section{Comparison to other studies of tonotopy in humans}

The results of this study are consistent with and extend other reports of tonotopic maps in the human auditory cortex. In

\footnotetext{
${ }^{10}$ Unnumbered FDRRs are analogous to unlabeled endpoints in that they fit the criteria for an FDRR but were not identified repeatably across subjects.
}

Talavage et al. (2000), we proposed that the tonotopic progression observed in evoked magnetic field studies of N100 (e.g., Cansino et al. 1994; Huotilainen et al. 1995; Lütkenhöner and Steinsträter 1998; Mühlnickel et al. 1998; Pantev et al. 1988; Yamamoto et al. 1988) corresponds to a tonotopic map connecting FDRRs 3 and 8. A progression was observed to connect endpoints $3^{\prime}$ and $8^{\prime}$, corresponding to these two FDRRs in five of six subjects. In all six subjects, a progression was observed between endpoints $2^{\prime}$ and $1^{\prime}$, corresponding to FDRRs 2 and 1, which may correspond to the tonotopic progression observed in the sustained field (SF) (Diesch and Luce 1997; Huotilainen et al. 1995; Pantev et al. 1994), in singleunit recordings (Howard et al. 1996) and in recent fMRI data ${ }^{11}$ (Formisano et al. 2002).

No consistent progression was observed between endpoints $1^{\prime}$ and $5^{\prime}$ corresponding to FDRRs 1 and 5, postulated to correspond to the middle-latency component $\mathrm{Pa}$ (Talavage et al. 2000). However, several recent fMRI studies did report a frequency progression with a location and orientation compatible with a tonotopic progression between FDRRs 1 and 5 (endpoints 1', 5') (Engelien et al. 2002; Formisano et al. 2002; Yang et al. 2000). Thus it is possible that FDRRs 1 and 5 are joined by a tonotopic map but that the techniques of the present study may not have been well suited to revealing it. In particular, our oblique slice plane, coupled with relatively coarse spatial resolution $(3.1 \times 3.1 \times 4 \mathrm{~mm})$ do not preclude partial volume averaging effects that could lead to a given voxel containing multiple, widely varying neuronal BFs. Analysis of such a voxel to determine the frequency to which the neurons are most sensitive is likely to produce either a mass average of the contained neuronal BFs or fail to identify a particular peak within the duration of the sweep. Note that our in-plane resolution is similar to the expected spatial extent of an octave on the surface of the cortex (e.g., Romani et al. 1982a,b).

\section{Comparison to non-human primate auditory cortex}

Similarities may exist between the tonotopic organization of human and monkey auditory koniocortex. We have observed two tonotopic maps on the medial half of $\mathrm{HG}$ that exhibit a mirror-image organization, sharing a lower-frequency endpoint $\left(1^{\prime}\right)$ with higher-frequency endpoints both anteromedial $\left(2^{\prime}\right)$ and posterolateral $\left(3^{\prime}\right)$ of the gyrus (Fig. 6D). These two progressions are proposed to be associated with the human koniocortex, representing KAm and KAlt of Galaburda and Sanides (1980). An organization of two tonotopic maps exhibiting a mirror-image organization across a lower-frequency boundary is also observed in the monkey koniocortex. In the macaque, $\mathrm{R}$ and AI have been observed to be tonotopically organized and to share a lower-frequency border in the middle of their combined anterior-posterior extent (Kosaki et al. 1997; Morel et al. 1993). Because the region of koniocortex defined in Galaburda and Pandya (1983) appears to correspond largely to the rostral area (R) (Morel et al. 1993), human KAm (medial AI) may be homologous to $\mathrm{R}$ in the monkey, whereas KAlt (lateral AI) may correspond to monkey AI. Given that the observed mirror-image organization in KAlt and KAm, our

\footnotetext{
11 The frequency progression between endpoints $1^{\prime}$ and $2^{\prime}$ is comparable in location to the more caudal (posterior) of the two maps reported by Formisano and colleagues.
} 
observed tonotopic organization within the human koniocortex is consistent with the monkey literature.

Further similarities may be observed between our human results and the monkey literature by examining the tonotopic organization in the parakoniocortex, adjacent and lateral to the koniocortex. We have proposed that two tonotopic maps exist in the anterior portion of $\mathrm{PaAi}$ with progressions originating in higher-frequency endpoints $5^{\prime}$ and $3^{\prime}$ exhibiting a mirrorimage organization across lower-frequency endpoint $6^{\prime}$. Endpoint $6^{\prime}$ is, in turn, located just posterolateral to the lowerfrequency boundary (endpoint $1^{\prime}$ ) of the two proposed koniocortical regions exhibiting a tonotopic organization (KAm and KAlt). PaAlt, the anatomical area in the monkey proposed to be homologous with PaAi (Galaburda and Pandya 1983), contains at least a portion of both the anterolateral (AL) and posterolateral (PL) anatomical areas (Morel et al. 1993). AL and PL have been observed in the macaque and owl monkey to contain mirror-image tonotopic maps, sharing a lower-frequency boundary that is located just lateral to the lower-frequency boundary of the tonotopic maps in R and AI (Kosaki et al. 1997; Morel and Kaas 1992)-i.e., a parallel structure of tonotopic organization is observed between the koniocortical and parakoniocortical areas. One difference between the monkey and human data is that the high-frequency ends of the tonotopic maps in $\mathrm{AL}$ and $\mathrm{R}$ are adjacent, whereas endpoints 5' and $2^{\prime}$ are not. In general, however, both the position relative to koniocortex and the orientation of the two maps attributed to the anterior portion of $\mathrm{PaAi}$ are similar to the position and orientation of the tonotopic maps observed in monkey PaAlt.

Not all of the tonotopic organization observed in the human auditory cortex is obviously related to that observed in the monkey. The tonotopic map proposed to be located in the posterior division of PaAi (endpoints $3^{\prime}$ and $8^{\prime}$ ) does not have an obvious homologous relationship with tonotopic areas described in the monkey. The posterior division of PaAi may be equivalent to the rhesus monkey caudal area (C) (Morel et al. 1993) or possibly a portion of the temporoparietal cortex (Tpt) (Galaburda and Pandya 1983) - a region of cortex believed to be related to speech processing in the human and demonstrated, in the macaque, to be used in the analysis of the temporal pattern of sounds (Leinonen et al. 1980). We are further unable to draw any conclusions regarding the similarity of tonotopic organization in human and monkey prokoniocortex due to a lack of physiological information for the monkey. ProA is proposed to be tonotopically organized with a progression of frequency sensitivity between endpoints $2^{\prime}$ and $7^{\prime}$, running almost parallel to that of KAm (endpoints $2^{\prime}$ and $1^{\prime}$ ). The corresponding monkey region, likely included in the rostromedial auditory area (RM) of Morel and co-workers (Morel and Kaas 1992; Morel et al. 1993), has not been thoroughly examined due to the difficulty of making depth electrode penetrations in this region (Imig et al. 1977; Merzenich and Brugge 1973).

The six tonotopic maps observed in the human may be assigned to parallel divisions of tonotopic areas, similar to those described in the macaque (Galaburda and Pandya 1983) based on cortico-cortical and thalamocortical connections. The parallel organization of tonotopic maps is similar to that observed by Kosaki et al. (1997) in the macaque in which a large lower-frequency region was centrally located, with higherfrequencies represented both anteriorly and posteriorly. Based on the parallel connectional organization of auditory areas in the macaque cortex and our presumed homologies between the human and monkey auditory cortex, we may readily assign the two anterior divisions of PaAi to the lateral belt, KAm and KAlt to the koniocortical core, and ProA to the medially located root division. The relatively high variability of the position of endpoint $8^{\prime}$ makes assignment of the posterior division of PaAi to a particular level of the auditory cortex impractical.

\section{Conclusions}

We have identified six progressions of frequency sensitivity on the human superior temporal lobe. The endpoints of these spatial progressions correspond well with seven of the eight FDRRs identified in the human auditory cortex (Talavage et al. 2000). Based on our previously described correspondence between FDRRs and underlying anatomically defined auditory areas, we conclude that five of these areas possess a tonotopic organization.

\section{A C KN O W LE D G MEN TS}

The authors thank Drs. Albert Galaburda, M. Christian Brown, and Randall Benson for time and graciousness in many insightful discussions. We also thank I. Sigalovsky and H. Penagos for comments and B. Norris and Dr. Bruce Fischl for assistance in the visual presentation of these data.

Present address of P. J. Ledden: Nova Medical, Wakefield, MA, 01880.

\section{G R A N T S}

This research was partially funded by National Institute on Deafness and Other Communication Disorders Grants P01DC-00119 and T32DC-00038-04 and a National Science Foundation Graduate Fellowship in electrical engineering.

\section{REFERENCES}

Bandettini PA, Jesmanowicz A, Wong EC, and Hyde JS. Processing strategies for functional MRI of the human brain. Magn Reson Med 30: 161-173, 1993.

Bandettini PA, Wong EC, Hinks RS, Tikofsky RS, and Hyde JS. Time course EPI of human brain function during task activation. Magn Reson Med 25: 390-397, 1992.

Bilecen D, Scheffler K, Schmid N, Tschopp K, and Seelig J. Tonotopic organization of human auditory cortex as detected by BOLD-FMRI. Hear Res 126: 19-27, 1998.

Cansino S, Williamson SJ, and Karron D. Tonotopic organization of human auditory association cortex. Brain Res 663: 38-50, 1994.

Clarey JC, Barone P, and Imig TJ. Functional organization of sound direction and sound pressure level in primary auditory cortex of the cat. J Neurophysiol 72: 2383-2405, 1994.

Dale AM and Sereno MI. Improved localization of cortical activity by combining EEG and MEG with MRI cortical surface reconstruction: a linear approach. J Cog Neurosci 5: 162-176, 1993.

Dale AM, Fischl B, and Sereno MI. Cortical surface-based analysis. I. Segmentation and surface reconstruction. Neuroimage 9: 179-194, 1999.

Diesch $\mathbf{E}$ and Luce T. Magnetic fields elicited by tones and vowel formants reveal tonotopy and nonlinear summation of cortical activation. Psychophysiology 34: 501-510, 1997.

Edmister WB, Talavage TM, Ledden PJ, and Weisskoff RM. Improved auditory cortex imaging using clustered volume acquisitions. Hum Brain Map 7: 88-97, 1999.

Engelien A, Yang Y, Engelien W, Zonana J, Stern E, and Silbersweig D. Physiological mapping of human auditory cortices with silent event-related fMRI technique. Neuroimage 16: 944-953, 2002.

Fischl B, Sereno MI, and Dale AM. Cortical surface-based analysis. II. Inflation, flattening and a surface-based coordinate system. Neuroimage 9: 195-207, 1999.

Fletcher NH. Acoustic Systems in Biology. New York: Oxford, 1992.

Formisano E, Kim DS, van de Moortele PF, Ugurbil K, and Goebel R. Mirror-symmetric tonotopic maps in the human primary auditory cortex 
revealed by event-related fMRI at 7 Tesla. $8^{\text {th }}$ International Conference on Functional Mapping of the Human Brain, June 2-6, 2002, Sendai, Japan.

Friston KJ, Ashburner J, Frith CD, Poline JB, Heather JD, and Frackowiak RSJ. Spatial registration and normalization of images. Hum Brain Map 2: 165-189, 1995.

Galaburda AM and Pandya DN. The intrinsic architectonic and connectional organization of the superior temporal region of the rhesus monkey. J Comp Neurol 221: 169-184, 1983.

Galaburda A and Sanides F. Cytoarchitectonic organization of the human auditory cortex. J Comp Neurol 190: 597-610, 1980.

Heil P, Rajan R, and Irvine DRF. Topographic representation of tone intensity along the isofrequency axis of cat primary auditory cortex. Hear Res 76: 188-202, 1994.

Howard MA III, Volkov IO, Abbas PJ, Damasio H, Ollendieck MC, and Granner MA. A chronic microelectrode investigation of the tonotopic organization of human auditory cortex. Brain Res 724: 260-264, 1996.

Howard MA, Volkov IO, Mirsky R, Garell PC, Noh MD, Granner M, Damasio H, Steinschneider M, Reale RA, Hind JE, and Brugge JF. Auditory cortex on the human posterior superior temporal gyrus. J Comp Neurol 416: 79-92, 2000.

Huotilainen M, Tiitinen H, Lavikainen J, Ilmoniemi RJ, Pekkonen E, Sinkkonen J, Laine P, and Näätänen R. Sustained fields of tones and glides reflect tonotopy of the auditory cortex. Neuroreport 6: 841-844, 1995 .

Imig TJ, Ruggero MA, Kitzes LM, Javel E, and Brugge JF. Organization of auditory cortex in the owl monkey (Aotus trivirgatus). J Comp Neurol 171: 111-128, 1977.

Kosaki H, Hashikawa T, He J, and Jones EG. Tonotopic organization of auditory cortical fields delineated by parvalbumin immunoreactivity in macaque monkeys. J Comp Neurol 386: 304-316, 1997.

Kwong KK, Belliveau JW, Chesler DA, Goldberg IE, Weisskoff RM, Poncelet BP, Kennedy DN, Hoppel BE, Cohen MS, Turner R, Cheng HM, Brady TJ, and Rosen BR. Dynamic magnetic resonance imaging of human brain activity during primary sensory stimulation. Proc Natl Acad Sci USA 89: 5675-5679, 1992.

Lauter JL, Herscovitch P, Formby C, and Raichle ME. Tonotopic organization in human auditory cortex revealed by positron emission tomography. Hear Res 20: 199-205, 1985.

Leinonen L, Hyvarinen J, and Sovijarvi ARA. Functional properties of neurons in the temporo-parietal association cortex of awake monkey. Exp Brain Res 39: 203-215, 1980.

Lockwood AH, Salvi RJ, Coad ML, Arnold SA, Wack DS, Murphy BW, and Burkard RF. The functional anatomy of the normal human auditory system: responses to 0.5 and $4.0 \mathrm{kHz}$ tones at varied intensities. Cereb Cortex 9: 65-76, 1999.

Lütkenhöner B and Steinsträter O. High-precision neuromagnetic study of the functional organization of the human auditory cortex. Audiol Neurootol 3: 191-213, 1998 .

Merzenich MM and Brugge JF. Representation of the cochlear partition on the superior temporal plane of the macaque monkey. Brain Res 50: 275-296, 1973.

Merzenich MM, Kaas JH, and Roth GL. Auditory cortex in the grey squirrel: tonotopic organization and architectonic fields. J Comp Neurol 166: 387-402, 1976.

Merzenich MM, Knight PL, and Roth GL. Representation of the cochlea within primary auditory cortex in the cat. J Neurophysiol 38: 321-349, 1975.

Middlebrooks JC and Pettigrew JD. Functional classes of neurons in primary auditory cortex of the cat distinguished by sensitivity to sound location. J Neurosci 1: 107-120, 1981.

Morel A, Garraghty PE, and Kaas JH. Tonotopic organization, architectonic fields, and connections of auditory cortex in macaque monkeys. J Comp Neurol 335: 437-459, 1993.

Morel A and Kaas JH. Subdivisions and connections of auditory cortex in owl monkeys. J Comp Neurol 318: 27-63, 1992.

Mühlnickel W, Elbert T, Taub E, and Flor H. Reorganization of auditory cortex in tinnitus. Proc Natl Acad. Sci. USA 95: 10340-10343, 1998.

Ogawa S, Lee TM, Kay AR, and Tank DW. Brain magnetic resonance imaging with contrast dependent on blood oxygenation. Proc Natl Acad Sci USA 87: 9868-9872, 1990.
Ogawa S, Tank DW, Menon R, Ellermann JM, Kim SG, Merkle H, and Ugurbil K. Intrinsic signal changes accompanying sensory stimulation: functional brain mapping using MRI. Proc Natl Acad Sci USA 89: 59515955, 1992.

Pandya DN. Anatomy of the auditory cortex. Rev Neurol (Paris) 151: 486494, 1995.

Pantev C, Hoke M, Lehnertz K, Lütkenhöner B, Anogianakis G, and Wittkowski W. Tonotopic organization of the human auditory cortex revealed by transient auditory evoked magnetic fields. Electroencephalogr Clin Neurophysiol 69: 160-170, 1988.

Pantev C, Eulitz C, Elbert T, and Hoke M. The auditory evoked sustained field: origin and frequency dependence. Electroencephalogr Clin Neurophysiol 90: 82-90, 1994.

Pantev C, Bertrand O, Eulitz C, Verkindt C, Hampson S, Schuierer G, and Elbert, T. Specific tonotopic organizations of different areas of human auditory cortex revealed by simultaneous magnetic and electric recordings. Electroencephalogr Clin Neurophysiol 94: 26-40, 1995.

Pantev C, Roberts LE, Elbert T, Ross B, and Wienbruch C. Tonotopic organization of the sources of human auditory steady-state responses. Hear Res 101: 62-74, 1996.

Phillips DP, Semple MN, Calford MB, and Kitzes LM. Level-dependent representation of stimulus frequency in cat primary auditory cortex. Exp Brain Res 102: 210-226, 1994.

Rademacher J, Caviness, Jr, VS, Steinmetz H, and Galaburda AM. Topographical variation of the human primary cortices: implications for neuroimaging, brain mapping and neurobiology. Cereb Cortex 3: 313-329, 1993.

Reale RA and Imig TJ. Tonotopic organization in auditory cortex of the cat. J Comp Neurol 192: 265-291, 1980.

Rivier F and Clarke S. Cytochrome oxidase, acetylcholinesterase, and NADPH-diaphorase staining in human supratemporal and insular cortex: evidence for multiple auditory areas. Neuroimage 6: 288-304, 1997.

Romani GL, Williamson SJ, and Kaufman L. Tonotopic organization of the human auditory cortex. Science 216: 1339-1340, 1982a.

Romani GL, Williamson SJ, Kaufman L, and Brenner D. Characterization of the human auditory cortex by the neuromagnetic method. Expr Brain Res 47: 381-393, 1982b.

Saad ZS, Ropella KM, Cox RW, and DeYoe EA. Analysis and use of fMRI response delays. Hum Brain Map 13: 74-93, 2001.

Schönwiesner M, von Cramon DY, and Rübsamen R. Is it tonotopy after all? Neuroimage 17: 1144-1161, 2002.

Schreiner CE and Urbas JV. Representation of amplitude modulation in the auditory cortex of the cat. II. Comparison between cortical fields. Hear Res 32: 49-63, 1988.

Sereno MI, Dale AM, Reppas JB, Kwong KK, Belliveau JW, Brady TJ, Rosen BR, and Tootell RBH. Borders of multiple visual areas in humans revealed by functional magnetic resonance imaging. Science 268: 889-893, 1995.

Talairach J and Tournoux P. Co-Planar Stereotaxic Atlas of the Human Brain. New York: Thieme Medical Publishers, 1988.

Talavage TM, Edmister WB, Ledden PJ, and Weisskoff RM. Quantitative assessment of auditory cortex response induced by imager acoustic noise. Hum Brain Map 7: 79-87, 1999.

Talavage TM, Ledden PJ, Benson RR, Rosen BR, and Melcher JR. Frequency-dependent responses exhibited by multiple regions in human auditory cortex. Hear Res 150: 225-244, 2000.

Wallace MN, Johnston PW, and Palmer AR. Histochemical identification of cortical areas in the auditory region of the human brain. Exp Brain Res 143: 499-508, 2002.

Wessinger CM, Buonocore MH, Kussmaul CL, and Mangun GR. Tonotopy in human auditory cortex examined with functional magnetic resonance imaging. Hum Brain Map 5: 18-25, 1997.

Yamamoto T, Williamson SJ, Kaufman L, Nicholson C, and Llinás R. Magnetic localization of neuronal activity in the human brain. Proc Natl Acad Sci USA 85: 8732-8736, 1988.

Yang Y, Engelien A, Engelien W, Xu S, Stern E, and Silbersweig DA. A silent event-related functional MRI technique for brain activation studies without interference of scanner acoustic noise. Magn Reson Med 43: 185190, 2000. 